\title{
Self-reported health behaviors, including sleep, correlate with doctor-informed medical conditions: data from the 2011 Health Related Behaviors Survey of U.S. Active Duty Military Personnel
}

\author{
Adela Hruby ${ }^{1,2}$, Harris R. Lieberman ${ }^{1}$ and Tracey J. Smith ${ }^{1 *}$
}

\begin{abstract}
Background: Health behaviors and cardiometabolic disease risk factors may differ between military and civilian populations; therefore, in U.S. active duty military personnel, we assessed relationships between demographic characteristics, self-reported health behaviors, and doctor-informed medical conditions.
\end{abstract}

Methods: Data were self-reported by 27,034 active duty military and Coast Guard personnel who responded to the 2011 Department of Defense Health Related Behaviors Survey. Multivariate linear and logistic regressions were used to estimate cross-sectional associations between (1) demographic characteristics (age, sex, service branch, marital status, children, race/ethnicity, pay grade) and self-reported behaviors (exercise, diet, smoking, alcohol, sleep); (2) demographic characteristics and doctor-informed medical conditions (hypertension, hypercholesterolemia, low high density lipoprotein (HDL) cholesterol, hyperglycemia) and overweight/obesity; and (3) behaviors and doctorinformed medical conditions.

Results: Among respondents (age $29.9 \pm 0.1$ years, 14.7\% female), females reported higher intake than men of fruit, vegetables, and dairy; those with higher education reported higher intakes of whole grains; those currently married and/or residing with children reported higher intake of starches. Older age and female sex were associated with higher odds (ORs 1.25 to 12.54 versus the youngest age group) of overweight/obesity. Older age and female sex were also associated with lower odds (ORs 0.29 to 0.65 versus male sex) of doctor-informed medical conditions, except for blood glucose, for which females had higher odds. Those currently married had higher odds of high cholesterol and overweight/obesity, and separated/divorced/widowed respondents had higher odds of high blood pressure and high cholesterol. Short sleep duration ( $<5$ versus $7-8 \mathrm{~h} / \mathrm{night}$ ) was associated with higher odds (ORs 1.36to 2.22) of any given doctor-informed medical condition. Strength training was associated with lower probability of high cholesterol, high triglycerides, and low HDL, and higher probability of overweight/obesity. Dietary factors were variably associated with doctor-informed medical conditions and overweight/obesity.

Conclusions: This study observed pronounced associations between health behaviors-especially sleep-and medical conditions, thus adding to evidence that sleep is a critical, potentially modifiable behavior within this population. When possible, adequate sleep should continue to be promoted as an important part of overall health and wellness throughout the military community.

Keywords: Army, Navy, Air force, Marine corps, Coast guard, Cardiometabolic risk, Exercise, Sleep, Survey

\footnotetext{
* Correspondence: tracey.smith10.civ@mail.mil

${ }^{1}$ Military Nutrition Division, US Army Research Institute of Environmental

Medicine, Natick, MA 01760, USA

Full list of author information is available at the end of the article
}

(c) The Author(s). 2018 Open Access This article is distributed under the terms of the Creative Commons Attribution 4.0 International License (http://creativecommons.org/licenses/by/4.0/), which permits unrestricted use, distribution, and reproduction in any medium, provided you give appropriate credit to the original author(s) and the source, provide a link to the Creative Commons license, and indicate if changes were made. The Creative Commons Public Domain Dedication waiver (http://creativecommons.org/publicdomain/zero/1.0/) applies to the data made available in this article, unless otherwise stated. 


\section{Background}

The U.S. Military maintains extensive records on the health of US Military personnel and their families via its healthcare system. However, information regarding health behaviors is not usually present in these records. Therefore, to systematically assess health behaviors among active duty personnel, the U.S. Department of Defense conducts a triennial Health Related Behaviors Survey (HRBS) of active duty military personnel [1], which assesses a range of self-reported health behaviors and risks, such as physical activity, diet, drug and alcohol use, posttraumatic stress, and doctor-informed medical conditions. The representative survey includes responses from participants in all branches of the Department of Defense [DoD] (Army, Navy, Marine Corps, and Air Force), as well as U.S. Coast Guard [USCG] members. The survey is voluntary and anonymous, and is used to guide the health-related policies in the Armed Forces. The HRBS provides data that are otherwise unavailable in this large subset of the U.S. population, approximately 1.3 million service members, since active duty military are excluded from national or state health-related surveys, such as the National Health and Nutrition Examination Survey (NHANES) or the Behavioral Risk Factor Surveillance System (BRFSS).

HRBS data have been used in a number of ways to study active duty and USCG personnel, for example, to identify whether they meet the Healthy People 2010 objectives [2], to describe mental health patterns and associations [3-5], and assess changes over time in self-reported overweight/ obesity [6, 7]. In depth analysis of demographic characteristics and health-related behaviors, and their association with self-reported health outcomes, may alter the manner in which military personnel are assessed and counseled for potential modifiable and non-modifiable demographic and behavioral risks. Furthermore, associations between health behaviors and disease risk factors may be different in military versus non-military populations due to differences in occupational demands and lifestyle characteristics. For example, deployment absences and frequent moves are sources of family stress that are more common in military than civilian families [8, 9]. Military personnel are required to meet standards of physical fitness and weight (or body composition) [10] and engage in regular physical training $[11,12]$. Military personnel are part of a workforce with institutionalized standards and a unique warrior culture and ethos not present in the civilian workforce. [13] The unique occupational demands frequently involve high risk activities (e.g., combat), which are an inherent part of military service [14]. Also in contrast to civilians, and of specific relevance to the present research, military personnel and their dependents are eligible to receive healthcare at little or no cost [9].

Therefore, the aims of this study were to examine relationships between generally unmodifiable sociodemographic factors (e.g., age, sex, race/ethnicity) and self-reported health behaviors (e.g., exercise and diet), as well as relationships between these sociodemographic factors and self-reported, doctor-informed medical conditions (i.e., hypertension, dyslipidemia, and hyperglycemia) and overweight/obesity. We also examined the relationships between these self-reported health behaviors and the medical conditions.

\section{Methods}

\section{Survey participants}

The 2011 HRBS was conducted by ICF International under contract with the Office of the Assistant Secretary of Defense for Health Affairs, Tricare Management Activity (TMA; now Defense Health Agency), and the USCG [15]. Primary data collection was approved by the Office of the Assistant Secretary of Defense for Health Affairs/ TRICARE Management Activity (OASD (HA)/TMA), Human Research Protection Office. Informed consent was required and obtained at the beginning of the HRBS, and responses were anonymous. For the present secondary data analyses, we obtained a de-identified data file from TMA through a data use agreement. Because the data were previously collected and de-identified, the protocol for the present study was deemed exempt (not human subject research) by both the U.S. Army Research Institute of Environmental Medicine (Natick, MA) and the Office of the Assistant Secretary of Defense for Health Affairs/ TRICARE Management Activity (OASD (HA)/TMA), Human Research Protection Office.

The sampling strategy, survey administration, and questionnaire format are extensively described in the initial report of the 2011 HRBS [15]. In brief, a stratified random sample was drawn from all non-deployed active duty Army, Navy, Marine Corps, Air Force, and USCG personnel. A second stratified random sample was drawn within each service branch using a combination of sex and pay grade as stratification variables. The resulting group was randomly divided into a group to whom invitations to take the survey were sent, as well as two other groups to use in case of a low response rate. The USCG used a different sampling method, dividing its population into two groups, one of which was sampled using geographic clustering while the other was sampled using stratification on work setting, sex, and pay grade. The eligible sample of potential respondents was $168,664(154,011$ from the DoD services and 14,653 from the USCG) [15].

Invitations to participate in the survey and reminders to complete it were distributed via email. Participants were allowed to opt out of taking the survey. The survey was available for 5 months after the initial invitations were sent in August 2011 (October 2011 for USCG). Those who did not have listed email addresses were mailed physical invitations. The response rate was $22 \%$ from the DoD services, and 37\% from the USCG, with a 
total of 39,877 usable, eligible responses [15]. Individuals who did not respond to questions on the variables of interest were excluded from the present analysis, for a final analytic sample size of 27,034. Characteristics of those included and excluded from the present analyses are provided in Additional file 1: Table S1.

All variables described below are derived from responses to the publicly available 2011 HRBS questionnaire [16].

\section{Sociodemographic variables}

Sociodemographic variables included in the present analysis were service branch, sex, age, race/ethnicity, education, pay grade, marital status, and presence of minor children living with the respondent. Specifically, respondents were asked to report service branch (Army, Navy, Marine Corps, Air Force, Coast Guard), sex (male, female), age $(<20,21-25$, $26-35,36-45,46-65$ years), education (up to high school or equivalent, some college without a degree, 2-year college degree, 4-year college degree, graduate education), race/ethnicity (non-Hispanic white, non-Hispanic black, Hispanic, and other/multiple race/ethnicity), pay grade (7 categories), current marital status (never married, married, separated/ divorced/widowed), and number of children $<18$ years living with the respondent at the duty station $(0, \geq 1$ children).

\section{Health behavior variables}

Health behaviors assessed included modifiable lifestyle factors: dietary intake, physical activity, cigarette smoking, alcohol intake, and sleep.

The 2011 HRBS questionnaire [16] asked about food intake during a given week, specifically the frequency of consumption of fruit (including "fresh, frozen, canned, or dried"), starchy vegetables (including "white potatoes, corn, peas"), vegetables (including "fresh, frozen, canned, cooked, or raw [not fried]"), whole grains (including "rye, whole-grain bread, brown or wild rice, whole-wheat pasta, oatmeal, etc."), dairy (including "milk, yogurt, cheese, etc."), lean protein (including "baked or broiled lean [low fat] meat, eggs, natural peanut butter, nuts, beans or legumes, tofu"), snack foods (including "potato chips, corn chips, pretzels"), sweets (including "chocolate, candy, cake, pie, breakfast bars, etc."), sugary drinks (including "juice, regular soda, Kool-Aid, Yoo-hoo, sports drinks, etc."), caffeinated drinks (including "coffee, tea, or energy drinks [Red Bull, Monster, 5-Hour Energy, Power Shots, etc.]"), and fried foods (including "French fries, fried chicken, donuts, etc."). Response choices for each food were: rarely/ never to $\geq 3$ times per day. Each reported instance of eating a given food was counted as one serving of that food. For example, if a respondent reported they ate fruit twice per day, it was coded as two servings of fruit per day.

Respondents were asked about the frequency (daily to "not at all in the past 30 days") and duration ( $\geq 60 \mathrm{~min} /$ day to "never in the past month") of moderate ("exertion that raises heart rate and breathing, but you should be able to carry on a conversation comfortably during the activity") and vigorous ("exertion that is high enough that you would find it difficult to carry on a conversation during the activity") physical activity and strength training. Responses were recoded into minutes per week and summed for total physical activity; moderate and vigorous exercise minutes were also summed for total aerobic physical activity.

Cigarette smoking was classified into never-smoked, former smoker (abstinent for $>6 \mathrm{mo}$ ), or current smoker based on responses to questions on lifetime cigarette smoking, last smoking occasion, current smoking, and cigarettes per day.

Alcohol intake (drinks/day in last month) was quantified based on responses to questions on the average frequency and amount of alcohol consumed in the last month.

Sleep (hrs/d) was coded based on reported sleep hours in a typical 24-h period in the previous week. Reported typical sleep hours $\leq 2$ or $\geq 16 \mathrm{~h} / \mathrm{d}$ over the previous week were considered non-habitual (i.e., rare or due to extenuating circumstances such as periodic training exercise, illness, etc.).

\section{Doctor-informed medical condition and overweight/ obesity variables}

Medical conditions in the present analysis included overweight/obesity, and 4 doctor-informed health conditions (hypertension, hypercholesterolemia, low HDL cholesterol, hyperglycemia).

Respondents were asked to report their height (feet and inches) and their weight (pounds). Body mass index (BMI) was calculated as the weight $(\mathrm{kg})$ divided by the square of the height $(\mathrm{m})$. BMI values $\leq 12$ or $\geq 50 \mathrm{~kg} / \mathrm{m}^{2}$ were considered implausible.

Respondents were asked whether they had been told by a doctor or other health care professional that they had high blood pressure, high blood sugar, high cholesterol, low HDL cholesterol ("low amounts of good cholesterol"), or high triglycerides ("blood fat"). Response choices were "No", "Yes, within the past 2 years," or "Yes, more than 2 years ago". Either yes response was coded as having the condition.

\section{Other covariates}

In addition to the above, we included weight history based on whether a respondent reported having had to lose weight to enter service and if so, how much (5-lb categories ranging from 0 to $\geq 30 \mathrm{lbs}$ ). Respondents were also asked whether they were currently enrolled in mandatory weight control program (yes, no). A yes response was considered a marker of history of overweight/obesity, irrespective of current reported (calculated) BMI. We also assessed history of deployment since September 11, 2001 (yes, no). 


\section{Statistical analysis}

The original survey incorporated sample weights applied post-stratification to compensate for lower response rates and disparate selection probabilities across strata and enable the sample to resemble the active duty population as a whole. Separate weights were applied for USCG members based on the mode of sampling used with the goal to reflect the entire population of the USCG [15]. In the present analysis, we used a restricted sample with complete responses on questions/variables of interest (exclusions noted above). Because responses may be missing at random, or missing completely at random, for systematic reasons (e.g., survey length), or for biased reasons (e.g., undesirable response), and because such reasons are unknown, we did not impute responses of non-completers from completer data. However, to maintain consistency of the restricted sample with the respondent sample, and thus, with the active duty population, we adjusted the original sample weights by multiplying the original weights of respondents within each stratum (service, sex, pay grade) by the number of completers in each stratum. These adjusted sample weights were used in the present analyses.

For binary dependent variables, logistic regression was used to estimate odds ratios (OR) and 95\% confidence intervals $(\mathrm{CI})$ of the outcome; for continuous dependent variables, linear regression was used to estimate least square means and standard errors of the dependent variable in categories of the independent variables. Survey procedures available in SAS (v9.3, Cary, North Carolina) designed to deal with complex survey designs and sampling weights were used. Statistical significance was set at $\alpha=0.05$.

The present analyses evaluated the following associations: 1) demographic characteristics with health behaviors; 2) demographic characteristics with doctor-informed medical conditions and overweight/obesity, and 3) health behaviors with medical conditions. Models of demographic characteristics predicting either health behaviors or medical conditions included all demographic characteristics simultaneously, and additionally adjusted for enrollment in weight loss program, weight loss history, and deployment history. There were 3 models of health behavior associations with medical conditions: model 1 included all behaviors simultaneously; model 2 additionally adjusted for all demographic characteristics; model 3 additionally adjusted for enrollment in weight loss program, weight loss history, and deployment history. Results from all models can be found in the Additional file 1; for simplicity we present only the results of the fully adjusted model in the main text.

Given the large number of health behaviors evaluated, we also conducted secondary exploratory factor analysis to assess possible patterns within the 17 behaviors of diet, exercise, sleep, alcohol, smoking, that may predict medical conditions. Four factors (named "Healthy diet," "Unhealthy diet," "Exercise," and "Bad habits"; Additional file 1: Table S2) were derived using a principal components approach, with varimax orthogonal rotation, retaining eigenvalues $\geq 1$. Factors were named based on factor loadings $>|0.20|$ and subsequently included in logistic regression models (models $1-3$, as above, where behaviors were replaced with factors) assessing their associations with medical conditions.

\section{Results}

Of the 27,034 respondents included in the analysis, 29, 17, 21, 19, and 14\%, were in the Air Force, Army, Marines, Navy, and USCG, respectively (Table 1). The weighted mean (SEM) age of respondents was 29.9 (0.1) years; weighted percentages showed $14.7 \%$ were female, $69.8 \%$ identified as non-Hispanic white, $8.6 \%$ as non-Hispanic black, $25.5 \%$ had at least a high school education, $37.2 \%$ had completed some college, $58.9 \%$ were married, and $39.9 \%$ had one or more children residing with them. Just over $10 \%$ reported having to lose some weight to join a service, but only $3 \%$ reported being currently enrolled in a weight control program. A majority of respondents (61.3\%) reported having been deployed at some point since September 11, 2001.

Weighted responses indicated low weekly intake of fried food, sweets, and snacks ( 3-4 ser/wk), moderate intake of starches and sugary drinks ( 5-6 ser/wk), and at least daily intake of fruit, vegetables, whole grains, dairy, lean meat, and caffeinated drinks ( 8-10 ser/wk). Respondents reported 175, 116, and $104 \mathrm{~min} / \mathrm{wk}$. of moderate exercise, vigorous exercise, and strength training, respectively. Nearly $60 \%$ of the weighted sample reported never smoking. Average intake of alcohol in the prior month was a third of drink per day, with $>20 \%$ of the weighted sample reporting no alcohol intake. Average sleep duration was $6.3 \mathrm{~h} / \mathrm{night}$, with $32 \%$ reporting $6-7 \mathrm{~h} /$ night-the largest sleep category.

Nearly $24 \%$ of the sample reported having at least one medical condition of five possible doctor-informed conditions: $13.0 \%$ reported high blood pressure; $12.7 \%$ high cholesterol; $5.5 \%$ high triglycerides; $6.1 \%$ low HDL cholesterol; and $1.5 \%$ high blood glucose. Nearly $63 \%$ of the sample categorized themselves as overweight or obese, and mean BMI was $26 \mathrm{~kg} / \mathrm{m}^{2}$.

\section{Associations of sociodemographic variables and health behaviors}

In fully-adjusted models, several sociodemographic characteristics were associated with health behaviors. Exercise (both aerobic and strength training) duration was longer in younger age groups, males, those in the Army or Marine Corps, service members with higher educational attainment, separated/divorced/widowed, or individuals without children present (Table 2). Alcohol intake was highest in those age 21-25 y, and lower in higher age groups, was highest in those in the Marine Corps, males, in those with 
Table 1 Characteristics of 27,034 respondents to the 2011 Health Risk Behavior Survey, by service branch

\begin{tabular}{|c|c|c|c|c|c|c|c|c|c|c|c|c|}
\hline \multirow[b]{2}{*}{ Characteristics $^{a}$} & \multicolumn{2}{|c|}{ Air Force } & \multicolumn{2}{|l|}{ Army } & \multicolumn{2}{|c|}{ Marine Corps } & \multicolumn{2}{|l|}{ Navy } & \multicolumn{2}{|c|}{ Coast Guard } & \multicolumn{2}{|c|}{ Total Sample } \\
\hline & $\mathrm{N}$ & $\begin{array}{l}\text { Mean } \pm \\
\text { SEM or } \%\end{array}$ & $\mathrm{~N}$ & $\begin{array}{l}\text { Mean } \pm \\
\text { SEM or } \%\end{array}$ & $\mathrm{~N}$ & $\begin{array}{l}\text { Mean } \pm \\
\text { SEM or } \%\end{array}$ & $\mathrm{~N}$ & $\begin{array}{l}\text { Mean } \pm \\
\text { SEM or } \%\end{array}$ & $\mathrm{~N}$ & $\begin{array}{l}\text { Mean } \pm \\
\text { SEM or } \%\end{array}$ & $\mathrm{~N}$ & $\begin{array}{l}\text { Mean } \pm \\
\text { SEM or } \%\end{array}$ \\
\hline \multicolumn{13}{|l|}{ Sociodemographic } \\
\hline Age, y & 7846 & $29.87 \pm 0.09$ & 4726 & $31.96 \pm 0.15$ & 5608 & $26.63 \pm 0.10$ & 5169 & $30.61 \pm 0.13$ & 3685 & $31.05 \pm 0.13$ & 27,034 & $29.86 \pm 0.06$ \\
\hline$<20 y$ & 513 & 6.7 & 121 & 4.38 & 466 & 12.44 & 107 & 3.75 & 74 & 2.46 & 1281 & 6.35 \\
\hline $20-\leq 25 y$ & 2245 & 29.3 & 638 & 21.49 & 1619 & 43.47 & 911 & 28.34 & 761 & 24.36 & 6174 & 30.03 \\
\hline $25-\leq 30 y$ & 1707 & 23.9 & 862 & 22.96 & 1195 & 21.07 & 1051 & 24.25 & 910 & 26.64 & 5725 & 23.58 \\
\hline $30-\leq 35$ y & 1169 & 15.8 & 851 & 17.94 & 875 & 10.54 & 977 & 17.14 & 759 & 20.87 & 4631 & 16.04 \\
\hline $35-\leq 40$ y & 1220 & 13.6 & 947 & 16.54 & 841 & 7.69 & 970 & 14.39 & 572 & 13.29 & 4550 & 12.98 \\
\hline$>40 y$ & 992 & 10.7 & 1307 & 16.70 & 612 & 4.78 & 1153 & 12.13 & 609 & 12.39 & 4673 & 11.02 \\
\hline Sex, female & 2816 & 19.8 & 1647 & 14.3 & 1572 & 7.2 & 1936 & 16.6 & 556 & 13.17 & 8527 & 14.7 \\
\hline \multicolumn{13}{|l|}{ Education } \\
\hline High school or equiv. & 1096 & 15.9 & 531 & 19.99 & 1736 & 43.78 & 891 & 26.61 & 791 & 23.42 & 5045 & 25.5 \\
\hline Some college & 2620 & 36.4 & 1435 & 37.34 & 2051 & 36.38 & 1553 & 35.62 & 1427 & 41.99 & 9086 & 37.2 \\
\hline 2-year college & 1608 & 19.0 & 656 & 12.51 & 420 & 5.84 & 757 & 11.88 & 391 & 10.37 & 3832 & 12.6 \\
\hline 4-year college & 862 & 9.5 & 819 & 15.30 & 816 & 9.18 & 852 & 12.08 & 481 & 12.46 & 3830 & 11.3 \\
\hline Beyond college & 1660 & 19.2 & 1285 & 14.86 & 585 & 4.83 & 1116 & 13.81 & 595 & 11.76 & 5241 & 13.4 \\
\hline \multicolumn{13}{|l|}{ Race/ethnicity } \\
\hline Non-Hispanic white & 5736 & 74.3 & 3062 & 66.8 & 3699 & 67.0 & 3325 & 63.0 & 2861 & 77.4 & 18,683 & 69.8 \\
\hline Non-Hispanic black & 686 & 7.6 & 719 & 12.0 & 542 & 8.1 & 680 & 11.4 & 135 & 3.4 & 2762 & 8.6 \\
\hline Hispanic & 804 & 10.2 & 564 & 13.1 & 983 & 17.8 & 592 & 13.3 & 412 & 12.1 & 3355 & 13.1 \\
\hline Other/multiple & 620 & 7.9 & 381 & 8.1 & 384 & 7.2 & 572 & 12.3 & 277 & 7.2 & 2234 & 8.5 \\
\hline \multicolumn{13}{|l|}{ Marital status } \\
\hline Never married & 2270 & 29.3 & 842 & 23.12 & 1559 & 39.75 & 1323 & 31.49 & 942 & 28.13 & 6936 & 30.7 \\
\hline Currently married & 4669 & 60.4 & 3126 & 64.44 & 3388 & 51.27 & 3138 & 57.27 & 2365 & 62.21 & 16,686 & 58.9 \\
\hline $\begin{array}{l}\text { Separated/divorced/ } \\
\text { widowed }\end{array}$ & 907 & 10.3 & 758 & 12.45 & 661 & 8.99 & 708 & 11.23 & 378 & 9.67 & 3412 & 10.5 \\
\hline Children, N & 7846 & $0.81 \pm 0.01$ & 4726 & $0.85 \pm 0.02$ & 5608 & $0.60 \pm 0.02$ & 5169 & $0.75 \pm 0.02$ & 3685 & $0.79 \pm 0.02$ & 27,034 & $0.76 \pm 0.01$ \\
\hline None & 4537 & 57.6 & 2496 & 57.31 & 3116 & 68.02 & 2769 & 60.14 & 2026 & 57.21 & 14,944 & 60.1 \\
\hline One or more & 3309 & 42.40 & 2230 & 42.69 & 2492 & 31.98 & 2400 & 39.87 & 1659 & 42.79 & 12,090 & 39.9 \\
\hline \multicolumn{13}{|l|}{ Pay grade ${ }^{b}$} \\
\hline E1-E4 & 2718 & 35.96 & 1205 & 47.48 & 1850 & 57.82 & 957 & 39.14 & 993 & 33.15 & 7723 & 42.7 \\
\hline E5-E6 & 2186 & 34.39 & 1084 & 24.88 & 1529 & 23.46 & 1572 & 34.70 & 1293 & 37.46 & 7664 & 30.9 \\
\hline E7-E9 & 1287 & 9.99 & 673 & 10.03 & 859 & 7.49 & 1069 & 8.89 & 481 & 10.79 & 4369 & 9.4 \\
\hline WO1-WO5 & - & - & 517 & 2.84 & 312 & 1.11 & 228 & 0.50 & 163 & 3.51 & 1220 & 1.3 \\
\hline O1-03 & 1052 & 11.3 & 485 & 8.90 & 564 & 6.66 & 763 & 9.80 & 400 & ' & 3259 & 9.3 \\
\hline O4-O10 & 603 & 8.34 & 762 & 5.87 & 494 & 3.45 & 580 & 6.97 & 355 & 6.14 & 2794 & 6.3 \\
\hline $\begin{array}{l}\text { Enrolled in current weight } \\
\text { control program }\end{array}$ & 145 & 1.83 & 145 & 3.82 & 143 & 2.71 & 203 & 3.92 & 108 & 3.26 & 744 & 3.0 \\
\hline \multicolumn{13}{|l|}{ Weight loss to join service } \\
\hline None & 7076 & 89.87 & 4232 & 87.99 & 5000 & 87.03 & 4734 & 90.84 & 3387 & 91.34 & 24,429 & 89.3 \\
\hline$<5 \mathrm{lbs}$ & 70 & 0.80 & 39 & 0.92 & 56 & 0.74 & 35 & 0.58 & 26 & 0.66 & 226 & 0.8 \\
\hline $5-9$ lbs & 186 & 2.22 & 143 & 3.03 & 156 & 2.38 & 102 & 1.93 & 74 & 2.22 & 661 & 2.3 \\
\hline 10-14 lbs & 201 & 2.75 & 119 & 2.50 & 139 & 2.93 & 123 & 2.21 & 59 & 1.67 & 641 & 2.5 \\
\hline $15-19$ lbs & 84 & 1.13 & 51 & 1.30 & 73 & 1.89 & 54 & 1.35 & 45 & 1.41 & 307 & 1.4 \\
\hline
\end{tabular}


Table 1 Characteristics of 27,034 respondents to the 2011 Health Risk Behavior Survey, by service branch (Continued)

\begin{tabular}{|c|c|c|c|c|c|c|c|c|c|c|c|c|}
\hline \multirow[b]{2}{*}{ Characteristics $^{\mathrm{a}}$} & \multicolumn{2}{|c|}{ Air Force } & \multicolumn{2}{|l|}{ Army } & \multicolumn{2}{|c|}{ Marine Corps } & \multicolumn{2}{|l|}{ Navy } & \multicolumn{2}{|c|}{ Coast Guard } & \multicolumn{2}{|c|}{ Total Sample } \\
\hline & $\mathrm{N}$ & $\begin{array}{l}\text { Mean } \pm \\
\text { SEM or } \%\end{array}$ & $\mathrm{~N}$ & $\begin{array}{l}\text { Mean } \pm \\
\text { SEM or } \%\end{array}$ & $\mathrm{~N}$ & $\begin{array}{l}\text { Mean } \pm \\
\text { SEM or } \%\end{array}$ & $\mathrm{~N}$ & $\begin{array}{l}\text { Mean } \pm \\
\text { SEM or } \%\end{array}$ & $\mathrm{~N}$ & $\begin{array}{l}\text { Mean } \pm \\
\text { SEM or } \%\end{array}$ & $\mathrm{~N}$ & $\begin{array}{l}\text { Mean } \pm \\
\text { SEM or } \%\end{array}$ \\
\hline 20-29 lbs & 108 & 1.45 & 48 & 1.23 & 73 & 1.76 & 59 & 1.27 & 38 & 1.05 & 326 & 1.4 \\
\hline$\geq 30 \mathrm{lbs}$ & 121 & 1.78 & 94 & 3.04 & 111 & 3.26 & 62 & 1.82 & 56 & 1.65 & 444 & 2.3 \\
\hline Deployed since 9/2001 & 4384 & 57.25 & 3587 & 69.25 & 3820 & 57.96 & 3949 & 70.22 & 1980 & 52.58 & 17,720 & 61.26 \\
\hline \multicolumn{13}{|l|}{ Health behaviors } \\
\hline \multicolumn{13}{|l|}{ Dietary intake (ser/wk) } \\
\hline Fruit & 7846 & $8.74 \pm 0.08$ & 4726 & $7.81 \pm 0.12$ & 5608 & $7.98 \pm 0.11$ & 5169 & $8.64 \pm 0.12$ & 3685 & $9.11 \pm 0.11$ & 27,034 & $8.45 \pm 0.05$ \\
\hline Starch & & $5.94 \pm 0.06$ & & $6.11 \pm 0.10$ & & $6.29 \pm 0.10$ & & $6.04 \pm 0.10$ & & $6.12 \pm 0.09$ & & $6.09 \pm 0.04$ \\
\hline Vegetables & & $9.75 \pm 0.08$ & & $9.12 \pm 0.12$ & & $8.68 \pm 0.11$ & & $9.54 \pm 0.12$ & & $10.20 \pm 0.11$ & & $9.44 \pm 0.05$ \\
\hline Whole grains & & $9.70 \pm 0.08$ & & $9.24 \pm 0.12$ & & $9.17 \pm 0.11$ & & $9.24 \pm 0.12$ & & $9.69 \pm 0.11$ & & $9.42 \pm 0.05$ \\
\hline Dairy & & $9.61 \pm 0.07$ & & $9.25 \pm 0.13$ & & $8.89 \pm 0.12$ & & $9.05 \pm 0.12$ & & $9.97 \pm 0.11$ & & $9.35 \pm 0.05$ \\
\hline Lean meat & & $9.81 \pm 0.07$ & & $9.41 \pm 0.13$ & & $9.48 \pm 0.12$ & & $9.40 \pm 0.12$ & & $10.12 \pm 0.11$ & & $9.64 \pm 0.05$ \\
\hline Snacks & & $3.66 \pm 0.05$ & & $3.68 \pm 0.09$ & & $4.17 \pm 0.09$ & & $3.89 \pm 0.09$ & & $3.84 \pm 0.08$ & & $3.84 \pm 0.04$ \\
\hline Sweets & & $3.61 \pm 0.05$ & & $3.75 \pm 0.09$ & & $3.80 \pm 0.09$ & & $3.85 \pm 0.09$ & & $3.66 \pm 0.08$ & & $3.73 \pm 0.04$ \\
\hline Sugary drinks & & $4.68 \pm 0.07$ & & $5.86 \pm 0.14$ & & $6.22 \pm 0.12$ & & $5.05 \pm 0.12$ & & $4.39 \pm 0.10$ & & $5.24 \pm 0.05$ \\
\hline Caffeinated drinks & & $7.38 \pm 0.08$ & & $8.47 \pm 0.14$ & & $7.66 \pm 0.13$ & & $8.02 \pm 0.13$ & & $9.25 \pm 0.13$ & & $8.01 \pm 0.05$ \\
\hline Fried food & & $2.72 \pm 0.04$ & & $2.85 \pm 0.08$ & & $3.26 \pm 0.08$ & & $3.04 \pm 0.08$ & & $2.66 \pm 0.06$ & & $2.90 \pm 0.03$ \\
\hline
\end{tabular}

Exercise (min/wk)

Moderate aerobic

Vigorous aerobic

Strength training

Smoking

Current

Never

Alcohol intake, drinks/d

Non-drinker

$<1 / 2$ drink/d

1/2-1 drink/d

$1-<2$ drinks/d

$2-<4$ drinks/d

$\geq 4$ drinks/d

Sleep, hrs/night

$$
\begin{aligned}
& <5 \mathrm{~h} / \text { night } \\
& 5-<6 \mathrm{~h} / \text { night } \\
& 6-<7 \mathrm{~h} / \text { night } \\
& 7-<8 \mathrm{~h} / \text { night } \\
& 8-<9 \mathrm{~h} / \text { night } \\
& 9-<10 \mathrm{~h} / \text { night } \\
& \geq 10 \mathrm{~h} / \text { night }
\end{aligned}
$$

Medical conditions

High blood pressure

High cholesterol
$110.8 \pm 1.2$

$97.8 \pm 1.3$

$1221 \quad 16.63$

$1337 \quad 17.00$

$5288 \quad 66.38$

$7846 \quad 0.20 \pm 0.01$

$1784 \quad 22.57$

$4932 \quad 62.39$

$978 \quad 12.69$

$107 \quad 1.64$

$34 \quad 0.52$

$11 \quad 0.19$

$7846 \quad 6.58 \pm 0.02$

$503 \quad 6.45$

$1158 \quad 14.49$

$2433 \quad 31.56$

$2196 \quad 28.01$

$1309 \quad 16.57$

$\begin{array}{ll}176 & 2.11\end{array}$

$71 \quad 0.81$

$887 \quad 11.7$

$965 \quad 12.1$

\begin{tabular}{ll}
945 & 26.88 \\
882 & 18.28 \\
2899 & 54.85 \\
4726 & $0.31 \pm 0.01$ \\
1072 & 23.25 \\
2564 & 54.06 \\
872 & 16.28 \\
152 & 4.29 \\
49 & 1.66 \\
17 & 0.47 \\
4726 & $6.05 \pm 0.03$ \\
616 & 15.10 \\
960 & 21.71 \\
1599 & 32.88 \\
967 & 18.71 \\
489 & 9.79 \\
58 & 1.03 \\
37 & 0.76 \\
\hline
\end{tabular}

$836 \quad 16.2$

$858 \quad 13.9$

$\begin{array}{ll}1317 & 30.47 \\ 1061 & 16.07 \\ 3230 & 53.45 \\ 5608 & 0.45 \pm 0.02 \\ 1127 & 19.81\end{array}$

$1127 \quad 1981$

$2941 \quad 50.07$

$1116 \quad 19.47$

$253 \quad 6.08$

$139 \quad 3.69$

$32 \quad 0.88$

$5608 \quad 6.14 \pm 0.03$

$674 \quad 13.51$

$1190 \quad 22.52$

$1835 \quad 31.87$

$1194 \quad 18.97$

$580 \quad 10.82$

$67 \quad 1.05$

$\begin{array}{ll}68 & 1.26\end{array}$

$759 \quad 12.5$

$659 \quad 8.2$
$998 \quad 17.22$

319258.15

$51690.32 \pm 0.01$

$1021 \quad 20.47$

296755.46

$998 \quad 18.73$

$124 \quad 3.11$

$47 \quad 1.74$

$12 \quad 0.47$

$51696.24 \pm 0.03$

$\begin{array}{ll}536 & 12.65\end{array}$

103120.06

$1740 \quad 31.74$

$1186 \quad 21.51$

$561 \quad 11.49$

$57 \quad 1.20$

$58 \quad 1.34$

$\begin{array}{ll}775 & 13.8\end{array}$

$989 \quad 15.3$

$\begin{array}{llll}675 & 19.87 & 5137 & 23.24 \\ 774 & 21.12 & 5052 & 17.64 \\ 2236 & 59.02 & 16,845 & 59.11 \\ 3685 & 0.31 \pm 0.01 & 27,034 & 0.31 \pm 0.0 \\ 527 & 14.76 & 5531 & 20.64 \\ 2150 & 58.44 & 15,554 & 56.53 \\ 851 & 22.22 & 4815 & 17.17 \\ 109 & 3.20 & 745 & 3.52 \\ 36 & 1.06 & 305 & 1.68 \\ 12 & 0.32 & 84 & 0.45\end{array}$

$3685 \quad 6.55 \pm 0.02$

$186 \quad 4.77$

$551 \quad 14.63$

$1278 \quad 34.35$

$1087 \quad 29.80$

$509 \quad 14.23$

$44 \quad 1.27$

$30 \quad 0.94$

$456 \quad 11.7$

$627 \quad 15.6$
$27,034 \quad 6.33 \pm 0.01$

$2515 \quad 10.36$

$4890 \quad 18.48$

$8885 \quad 32.27$

$6630 \quad 23.54$

$3448 \quad 12.92$

$402 \quad 1.42$

$264 \quad 1.01$ 
Table 1 Characteristics of 27,034 respondents to the 2011 Health Risk Behavior Survey, by service branch (Continued)

\begin{tabular}{|c|c|c|c|c|c|c|c|c|c|c|c|c|}
\hline \multirow[b]{2}{*}{ Characteristics $^{\mathrm{a}}$} & \multicolumn{2}{|c|}{ Air Force } & \multicolumn{2}{|l|}{ Army } & \multicolumn{2}{|c|}{ Marine Corps } & \multicolumn{2}{|l|}{ Navy } & \multicolumn{2}{|c|}{ Coast Guard } & \multicolumn{2}{|c|}{ Total Sample } \\
\hline & $\mathrm{N}$ & $\begin{array}{l}\text { Mean } \pm \\
\text { SEM or } \%\end{array}$ & $\mathrm{~N}$ & $\begin{array}{l}\text { Mean } \pm \\
\text { SEM or } \%\end{array}$ & $\mathrm{~N}$ & $\begin{array}{l}\text { Mean } \pm \\
\text { SEM or } \%\end{array}$ & $\mathrm{~N}$ & $\begin{array}{l}\text { Mean } \pm \\
\text { SEM or } \%\end{array}$ & $\mathrm{~N}$ & $\begin{array}{l}\text { Mean } \pm \\
\text { SEM or } \%\end{array}$ & $\mathrm{~N}$ & $\begin{array}{l}\text { Mean } \pm \\
\text { SEM or } \%\end{array}$ \\
\hline High triglycerides & 454 & 5.9 & 324 & 5.2 & 179 & 2.1 & 470 & 7.4 & 318 & 7.7 & 1745 & 5.5 \\
\hline Low HDL cholesterol & 474 & 6.1 & 416 & 6.7 & 219 & 2.7 & 478 & 7.7 & 334 & 8.1 & 1921 & 6.1 \\
\hline High blood glucose & 92 & 1.1 & 110 & 1.8 & 78 & 1.1 & 151 & 2.4 & 68 & 1.6 & 499 & 1.5 \\
\hline Body mass index, $\mathrm{kg} / \mathrm{m}^{2}$ & 7846 & $25.74 \pm 0.04$ & 4726 & $26.57 \pm 0.07$ & 5608 & $25.36 \pm 0.05$ & 5169 & $26.42 \pm 0.06$ & 3685 & $26.33 \pm 0.06$ & 27,034 & $26.02 \pm 0.03$ \\
\hline Overweight/obese & 4309 & 58.2 & 3128 & 67.8 & 3005 & 58.2 & 3358 & 66.0 & 2492 & 67.6 & 16,292 & 62.6 \\
\hline Total conditions, N & 7846 & $0.37 \pm 0.01$ & 4726 & $0.44 \pm 0.01$ & 5608 & $0.27 \pm 0.01$ & 5169 & $0.47 \pm 0.02$ & 3685 & $0.45 \pm 0.02$ & 27,034 & $0.39 \pm 0.01$ \\
\hline$\geq 1$ condition(s) & 1749 & 22.3 & 1528 & 27.1 & 1263 & 18.5 & 1607 & 26.5 & 1047 & 26.1 & 7194 & 23.7 \\
\hline
\end{tabular}

a Characteristics are weighted to representative populations

${ }^{\mathrm{b}}$ Pay grade E denotes Enlisted, W denotes Warrant Officer, and O denotes Officer

lower educational attainment, non-Hispanic whites, and was lowest among those currently married and those with children present. Average sleep time was longest in the youngest age category and in those in the Air Force or Coast Guard. Current smoking was less likely in the older age categories, among those in the Army or Marine Corps, in the currently or previously married, and was more likely in males, in those with higher educational attainment, and in non-Hispanic blacks.

Dietary intake of all food groups except vegetables was highest in the youngest age category, and was lower in progressively older age categories (Table 3). Differences in food intake (e.g., starch and fried food) between service branches were small $(<2 \mathrm{ser} / \mathrm{wk})$ or statistically the same across all sociodemographic characteristics. Females reported higher intake than males of fruit, vegetables, and dairy, and lower intake of sugary drinks and fried foods. Those with higher education reported higher intakes of fruit, vegetables, whole grains, and dairy than those with lower education. Those who reported being currently married or having children present reported higher intake of fruit, starches, vegetables, whole grains, and dairy relative to their not married or childless counterparts.

\section{Demographic variables associated with medical conditions}

In the fully-adjusted models, specific sociodemographic characteristics were associated with doctor-informed medical conditions and overweight/obesity (Table 4; Additional file 1: Table S3). Odds of all conditions were higher amongst older age compared to the youngest age category, and in males compared to females, except for high blood glucose for which females had higher odds than males.

There were no discernible patterns in reported doctor-informed medical conditions and overweight/ obesity between the service branches. However, those in the Navy had markedly higher odds of high blood glucose ( $45 \%$ higher odds compared with the Army) and those in the Coast Guard or Navy had markedly higher odds of high triglycerides ( 54-59\% higher odds compared with the Army) (Table 4).

Higher educational attainment tended to be associated with incrementally higher odds of high cholesterol, low HDL cholesterol, high triglycerides, and with lower odds of overweight/obesity, associations which were not accounted for by simultaneously adjusting for age. For example, relative to those with a high school education or equivalent, those with some college, 2 years of college, 4 years of college, and those with education beyond college, had 1.25, $1.50,1.52$, and 1.92 times the odds, respectively, of having low HDL cholesterol (Table 4).

Compared to non-Hispanic whites, non-Hispanic blacks had $68 \%$ higher odds of high blood pressure, $36 \%$ higher odds of overweight/obesity, $42 \%$ lower odds of high triglycerides, and 22\% lower odds of HDL. Hispanic personnel had 39\% higher odds of high blood glucose, and $43 \%$ higher odds of overweight/obesity relative to non-Hispanic whites (Table 4).

Currently married respondents, compared to those who were never married, had significantly higher odds of high cholesterol and overweight/obesity, while those who were separated/divorced/widowed had significantly higher odds of high blood pressure. Having children present in the home was also associated with having a medical condition: compared to those without children living with them, those with children had 24,32, 64, and 23\% higher odds of high triglycerides, low HDL cholesterol, high blood glucose, and overweight/obesity, respectively (Table 4).

\section{Behaviors associated doctor-informed medical conditions and overweight/obesity}

Of the 17 health behaviors assessed, the health behavior most consistently associated with every doctor-informed medical condition and overweight/obesity was shorter duration of sleep. In fully adjusted models, compared to 
Table 2 Associations between sociodemographic characteristics and health behaviors (i.e., exercise, alcohol, sleep, and smoking) ${ }^{a}$

\begin{tabular}{|c|c|c|c|c|c|c|c|}
\hline \multirow[b]{2}{*}{$\begin{array}{l}\text { Demographic } \\
\text { Characteristic } \\
\end{array}$} & \multirow[b]{2}{*}{ Categories } & \multicolumn{5}{|l|}{ Mean \pm SE } & \multirow{2}{*}{$\begin{array}{l}\text { OR }(95 \% \mathrm{Cl}) \\
\text { Current smoking }\end{array}$} \\
\hline & & $\begin{array}{l}\text { Moderate exercise } \\
\text { (min/wk) }\end{array}$ & $\begin{array}{l}\text { Vigorous exercise } \\
\text { (min/wk) }\end{array}$ & $\begin{array}{l}\text { Strength training } \\
\text { (min/wk) }\end{array}$ & $\begin{array}{l}\text { Alcohol } \\
\text { (drinks/d) }\end{array}$ & $\begin{array}{l}\text { Sleep } \\
\text { (hrs/night) }\end{array}$ & \\
\hline \multirow[t]{5}{*}{ Age } & $\leq 20$ yrs. (ref.) & $171.9 \pm 8.33$ & $122.5 \pm 8.28$ & $104.3 \pm 7.71$ & $0.05 \pm 0.03$ & $6.5 \pm 0.08$ & 1 \\
\hline & $21-25$ yrs & $155.1 \pm 6.84$ & $111.7 \pm 7.24$ & $101.7 \pm 6.33$ & $0.31 \pm 0.02$ & $6.3 \pm 0.06$ & $0.68(0.57-0.80)$ \\
\hline & $26-35$ yrs & $150.7 \pm 6.46$ & $104.2 \pm 7.00$ & $88.8 \pm 6.00$ & $0.27 \pm 0.02$ & $6.2 \pm 0.05$ & $0.50(0.41-0.60)$ \\
\hline & $36-45$ yrs & $137.4 \pm 6.47$ & $89.6 \pm 7.02$ & $70.4 \pm 6.00$ & $0.22 \pm 0.02$ & $6.1 \pm 0.05$ & $0.52(0.42-0.64)$ \\
\hline & $46+y r s$ & $133.3 \pm 7.41$ & $81.3 \pm 7.57$ & $62.1 \pm 6.51$ & $0.20 \pm 0.02$ & $6.1 \pm 0.06$ & $0.46(0.36-0.59)$ \\
\hline \multirow[t]{5}{*}{ Service } & Army (ref.) & $178.0 \pm 6.78$ & $119.1 \pm 7.24$ & $96.6 \pm 6.28$ & $0.22 \pm 0.02$ & $6.0 \pm 0.06$ & 1 \\
\hline & Air Force & $135.0 \pm 6.45$ & $97.6 \pm 7.00$ & $81.9 \pm 5.99$ & $0.11 \pm 0.02$ & $6.5 \pm 0.05$ & $1.66(1.50-1.83)$ \\
\hline & Coast Guard & $137.4 \pm 6.79$ & $88.3 \pm 7.20$ & $77.6 \pm 6.23$ & $0.20 \pm 0.02$ & $6.5 \pm 0.06$ & $1.46(1.30-1.63)$ \\
\hline & Marine Corps & $155.5 \pm 6.74$ & $112.1 \pm 7.20$ & $97.0 \pm 6.28$ & $0.32 \pm 0.02$ & $6.0 \pm 0.06$ & $1.04(0.92-1.17)$ \\
\hline & Navy & $142.5 \pm 6.70$ & $92.2 \pm 7.14$ & $74.3 \pm 6.19$ & $0.22 \pm 0.02$ & $6.2 \pm 0.06$ & $1.18(1.05-1.32)$ \\
\hline \multirow[t]{2}{*}{ Sex } & Female (ref.) & $141.6 \pm 6.45$ & $89.6 \pm 6.99$ & $66.1 \pm 5.96$ & $0.14 \pm 0.02$ & $6.3 \pm 0.05$ & 1 \\
\hline & Male & $157.7 \pm 6.42$ & $114.1 \pm 6.98$ & $104.8 \pm 5.98$ & $0.29 \pm 0.02$ & $6.2 \pm 0.05$ & $1.24(1.16-1.33)$ \\
\hline \multirow[t]{5}{*}{ Education } & High school or equiv. (ref.) & $139.6 \pm 6.82$ & $91.5 \pm 7.26$ & $79.3 \pm 6.33$ & $0.27 \pm 0.02$ & $6.2 \pm 0.06$ & 1 \\
\hline & Some college & $149.4 \pm 6.62$ & $99.9 \pm 7.12$ & $84.7 \pm 6.12$ & $0.22 \pm 0.02$ & $6.1 \pm 0.06$ & $1.26(1.15-1.38)$ \\
\hline & 2-year college & $145.6 \pm 6.88$ & $101.4 \pm 7.31$ & $87.4 \pm 6.36$ & $0.20 \pm 0.02$ & $6.2 \pm 0.06$ & $1.65(1.47-1.85)$ \\
\hline & 4-year college & $160.0 \pm 6.87$ & $106.5 \pm 7.26$ & $89.8 \pm 6.31$ & $0.18 \pm 0.02$ & $6.3 \pm 0.06$ & $2.73(2.38-3.13)$ \\
\hline & Beyond college & $153.8 \pm 6.93$ & $110.0 \pm 7.32$ & $86.0 \pm 6.34$ & $0.18 \pm 0.02$ & $6.4 \pm 0.06$ & $3.28(2.79-3.84)$ \\
\hline \multirow[t]{4}{*}{ Race/ethnicity } & Non-Hispanic white (ref.) & $155.0 \pm 6.36$ & $101.4 \pm 6.93$ & $74.8 \pm 5.91$ & $0.27 \pm 0.02$ & $6.3 \pm 0.05$ & 1 \\
\hline & Non-Hispanic black & $144.3 \pm 6.89$ & $95.7 \pm 7.34$ & $90.0 \pm 6.39$ & $0.16 \pm 0.02$ & $6.2 \pm 0.06$ & $3.57(3.11-4.09)$ \\
\hline & Hispanic & $151.6 \pm 6.86$ & $108.8 \pm 7.30$ & $91.4 \pm 6.39$ & $0.22 \pm 0.02$ & $6.3 \pm 0.06$ & $1.73(1.56-1.92)$ \\
\hline & Other/multiple & $147.9 \pm 7.12$ & $101.5 \pm 7.47$ & $85.6 \pm 6.58$ & $0.18 \pm 0.02$ & $6.1 \pm 0.06$ & $1.17(1.04-1.32)$ \\
\hline \multirow[t]{3}{*}{ Marital status } & Never married (ref.) & $144.6 \pm 6.62$ & $101.8 \pm 7.11$ & $83.4 \pm 6.14$ & $0.23 \pm 0.02$ & $6.3 \pm 0.06$ & 1 \\
\hline & Currently married & $150.3 \pm 6.36$ & $99.4 \pm 6.94$ & $80.6 \pm 5.93$ & $0.15 \pm 0.02$ & $6.4 \pm 0.05$ & $0.85(0.77-0.93)$ \\
\hline & Separated/divorced/ widowed & $154.1 \pm 6.98$ & $104.4 \pm 7.32$ & $92.4 \pm 6.44$ & $0.25 \pm 0.02$ & $6.0 \pm 0.06$ & $0.59(0.52-0.67)$ \\
\hline \multirow[t]{2}{*}{ Children } & 0 children (ref.) & $154.0 \pm 6.37$ & $105.2 \pm 6.94$ & $89.3 \pm 5.93$ & $0.24 \pm 0.02$ & $6.3 \pm 0.05$ & 1 \\
\hline & $1+$ children & $145.3 \pm 6.56$ & $98.5 \pm 7.07$ & $81.5 \pm 6.08$ & $0.18 \pm 0.02$ & $6.2 \pm 0.05$ & $0.89(0.83-0.96)$ \\
\hline \multirow[t]{6}{*}{ Pay grade } & E1-E4 (ref.) & $176.1 \pm 2.82$ & $107.1 \pm 2.36$ & $99.5 \pm 2.40$ & $0.16 \pm 0.01$ & $6.3 \pm 0.03$ & 1 \\
\hline & E5-E6 & $167.7 \pm 2.73$ & $108.4 \pm 2.30$ & $97.9 \pm 2.33$ & $0.18 \pm 0.01$ & $6.2 \pm 0.03$ & $0.82(0.75-0.91)$ \\
\hline & E7-E9 & $165.3 \pm 3.14$ & $111.0 \pm 2.61$ & $97.3 \pm 2.66$ & $0.24 \pm 0.01$ & $6.3 \pm 0.03$ & $0.93(0.82-1.06)$ \\
\hline & W01-WO5 & $161.5 \pm 5.60$ & $102.3 \pm 4.35$ & $87.9 \pm 4.30$ & $0.24 \pm 0.02$ & $6.5 \pm 0.05$ & $1.12(0.91-1.37)$ \\
\hline & $\mathrm{O} 1-\mathrm{O} 3$ & $150.3 \pm 3.64$ & $102.7 \pm 3.06$ & $87.0 \pm 3.10$ & $0.22 \pm 0.01$ & $6.5 \pm 0.03$ & $1.72(1.48-2.00)$ \\
\hline & O4-O10 & $143.3 \pm 4.28$ & $101.9 \pm 3.57$ & $82.3 \pm 3.42$ & $0.30 \pm 0.01$ & $6.4 \pm 0.04$ & $2.24(1.84-2.73)$ \\
\hline
\end{tabular}

${ }^{a}$ Reference categories are indicated for tests of differences between means or for odds ratios. All differences from the reference category are statistically significant $(P<0.05)$, except for means or odds ratios in bold. Linear regressions to estimate least square means, and logistic regressions to estimate odds ratios were adjusted for sample weights. Multivariate analyses included all demographic characteristics simultaneously

sleeping 7-8 h/night, sleeping $<5 \mathrm{~h} /$ night was associated with 1.36 (95\% CI 1.18-1.57, for overweight/obesity) to up to 2.22 (95\% CI 1.89-2.61, for hypertension) times the odds of having a medical condition (Fig. 1a-f; Additional file 1: Table S4). Odds of most conditions also tended to be higher at sleep durations $<6 \mathrm{~h} /$ night, relative to $7-8 \mathrm{~h} /$ night. Sleep durations of $8 \mathrm{~h} /$ night or longer were not statistically different from 7 to $8 \mathrm{~h} /$ night.
In addition, in fully adjusted models, strength training was associated with $2-5 \%$ lower odds of lipid-related conditions (i.e., high cholesterol, high triglycerides, and low HDL) per $30 \mathrm{~min} / \mathrm{wk}$. increment, as well as high blood pressure, but slightly higher odds of overweight/ obesity (4\% per $30 \mathrm{~min} / \mathrm{wk}$. increment). Neither vigorous nor moderate exercise was consistently associated with medical conditions. Compared with never-smoking, 
Table 3 Means of dietary intake by sociodemographic characteristics ${ }^{a}$

\begin{tabular}{|c|c|c|c|c|c|c|c|c|c|c|}
\hline \multirow{2}{*}{ Characteristic } & \multirow[b]{2}{*}{ Categories } & \multicolumn{9}{|c|}{ Servings/week (mean \pm SE) } \\
\hline & & Fruit & Starch & Vegetables & $\begin{array}{l}\text { Whole } \\
\text { grains }\end{array}$ & Dairy & Sweets & $\begin{array}{l}\text { Sugary } \\
\text { drinks }\end{array}$ & $\begin{array}{l}\text { Caffeinated } \\
\text { drinks }\end{array}$ & $\begin{array}{l}\text { Fried } \\
\text { food }\end{array}$ \\
\hline \multirow[t]{5}{*}{ Age } & $\leq 20$ yrs. (ref.) & $9.5 \pm 0.41$ & $6.3 \pm 0.35$ & $10.7 \pm 0.40$ & $10.9 \pm 0.46$ & $10.5 \pm 0.67$ & $4.7 \pm 0.26$ & $4.9 \pm 0.36$ & $5.7 \pm 0.33$ & $3.3 \pm 0.23$ \\
\hline & $21-25$ yrs & $9.0 \pm 0.34$ & $5.7 \pm 0.29$ & $10.6 \pm 0.33$ & $10.3 \pm 0.40$ & $10.0 \pm 0.63$ & $4.0 \pm 0.18$ & $4.2 \pm 0.26$ & $6.0 \pm 0.24$ & $2.7 \pm 0.14$ \\
\hline & $26-35$ yrs & $8.9 \pm 0.32$ & $5.5 \pm 0.27$ & $10.9 \pm 0.31$ & $9.8 \pm 0.39$ & $9.3 \pm 0.62$ & $3.8 \pm 0.16$ & $3.7 \pm 0.24$ & $7.0 \pm 0.21$ & $2.4 \pm 0.12$ \\
\hline & $36-45$ yrs & $8.3 \pm 0.33$ & $5.0 \pm 0.27$ & $10.4 \pm 0.32$ & $8.8 \pm 0.39$ & $8.2 \pm 0.62$ & $3.3 \pm 0.16$ & $3.3 \pm 0.24$ & $8.1 \pm 0.21$ & $2.1 \pm 0.12$ \\
\hline & $46+y r s$ & $8.3 \pm 0.37$ & $4.4 \pm 0.30$ & $10.4 \pm 0.36$ & $8.2 \pm 0.42$ & $7.7 \pm 0.64$ & $3.1 \pm 0.20$ & $2.8 \pm 0.28$ & $9.0 \pm 0.31$ & $1.8 \pm 0.14$ \\
\hline \multirow[t]{5}{*}{ Service } & Army (ref.) & $8.1 \pm 0.34$ & $5.5 \pm 0.28$ & $10.2 \pm 0.33$ & $9.5 \pm 0.40$ & $9.2 \pm 0.63$ & $3.9 \pm 0.18$ & $4.6 \pm 0.26$ & $7.4 \pm 0.23$ & $2.5 \pm 0.14$ \\
\hline & Air Force & $8.8 \pm 0.33$ & $5.2 \pm 0.27$ & $10.6 \pm 0.31$ & $9.7 \pm 0.39$ & $9.2 \pm 0.62$ & $3.6 \pm 0.16$ & $3.5 \pm 0.24$ & $6.3 \pm 0.21$ & $2.4 \pm 0.12$ \\
\hline & Coast Guard & $9.5 \pm 0.34$ & $5.4 \pm 0.28$ & $11.2 \pm 0.33$ & $9.9 \pm 0.40$ & $9.7 \pm 0.63$ & $3.8 \pm 0.17$ & $3.1 \pm 0.25$ & $7.7 \pm 0.24$ & $2.4 \pm 0.13$ \\
\hline & Marine Corps & $8.6 \pm 0.34$ & $5.4 \pm 0.28$ & $10.2 \pm 0.33$ & $9.3 \pm 0.40$ & $8.6 \pm 0.63$ & $3.7 \pm 0.17$ & $4.0 \pm 0.26$ & $7.2 \pm 0.23$ & $2.5 \pm 0.14$ \\
\hline & Navy & $9.0 \pm 0.34$ & $5.4 \pm 0.28$ & $10.7 \pm 0.33$ & $9.5 \pm 0.40$ & $9.0 \pm 0.63$ & $3.9 \pm 0.17$ & $3.7 \pm 0.25$ & $7.1 \pm 0.23$ & $2.6 \pm 0.14$ \\
\hline \multirow[t]{2}{*}{ Sex } & Female (ref.) & $9.3 \pm 0.33$ & $4.8 \pm 0.27$ & $11.0 \pm 0.31$ & $9.4 \pm 0.39$ & $9.3 \pm 0.62$ & $3.8 \pm 0.16$ & $2.9 \pm 0.24$ & $6.7 \pm 0.21$ & $2.0 \pm 0.12$ \\
\hline & Male & $8.3 \pm 0.32$ & $6.0 \pm 0.27$ & $10.2 \pm 0.31$ & $9.8 \pm 0.39$ & $8.9 \pm 0.62$ & $3.7 \pm 0.16$ & $4.6 \pm 0.24$ & $7.6 \pm 0.20$ & $3.0 \pm 0.12$ \\
\hline \multirow[t]{5}{*}{ Education } & $\begin{array}{l}\text { High school or } \\
\text { equiv. (ref.) }\end{array}$ & $8.0 \pm 0.34$ & $5.2 \pm 0.29$ & $9.8 \pm 0.33$ & $8.7 \pm 0.40$ & $8.8 \pm 0.63$ & $3.9 \pm 0.18$ & $5.0 \pm 0.26$ & $7.6 \pm 0.24$ & $2.8 \pm 0.14$ \\
\hline & Some college & $8.5 \pm 0.33$ & $5.4 \pm 0.28$ & $10.4 \pm 0.32$ & $9.3 \pm 0.39$ & $9.1 \pm 0.62$ & $3.5 \pm 0.16$ & $4.0 \pm 0.24$ & $7.2 \pm 0.22$ & $2.6 \pm 0.13$ \\
\hline & 2-year college & $8.9 \pm 0.35$ & $5.6 \pm 0.29$ & $10.6 \pm 0.34$ & $9.7 \pm 0.40$ & $9.1 \pm 0.63$ & $3.7 \pm 0.18$ & $3.4 \pm 0.26$ & $7.3 \pm 0.25$ & $2.4 \pm 0.14$ \\
\hline & 4-year college & $9.2 \pm 0.34$ & $5.3 \pm 0.29$ & $10.8 \pm 0.33$ & $10.0 \pm 0.40$ & $9.3 \pm 0.63$ & $4.0 \pm 0.18$ & $3.4 \pm 0.26$ & $6.8 \pm 0.24$ & $2.4 \pm 0.14$ \\
\hline & Beyond college & $9.5 \pm 0.35$ & $5.4 \pm 0.29$ & $11.4 \pm 0.34$ & $10.3 \pm 0.40$ & $9.4 \pm 0.63$ & $3.9 \pm 0.18$ & $3.1 \pm 0.26$ & $7.0 \pm 0.25$ & $2.2 \pm 0.14$ \\
\hline \multirow[t]{4}{*}{$\begin{array}{l}\text { Race/ } \\
\text { ethnicity }\end{array}$} & $\begin{array}{l}\text { Non-Hispanic white } \\
\text { (ref.) }\end{array}$ & $9.0 \pm 0.32$ & $5.6 \pm 0.27$ & $11.3 \pm 0.31$ & $10.2 \pm 0.38$ & $10.4 \pm 0.62$ & $3.7 \pm 0.15$ & $3.7 \pm 0.23$ & $9.3 \pm 0.20$ & $2.2 \pm 0.12$ \\
\hline & Non-Hispanic black & $8.5 \pm 0.35$ & $5.3 \pm 0.29$ & $10.0 \pm 0.34$ & $8.9 \pm 0.41$ & $8.1 \pm 0.63$ & $4.1 \pm 0.19$ & $4.8 \pm 0.27$ & $4.6 \pm 0.23$ & $3.0 \pm 0.16$ \\
\hline & Hispanic & $8.6 \pm 0.34$ & $5.0 \pm 0.29$ & $10.0 \pm 0.33$ & $9.2 \pm 0.40$ & $9.1 \pm 0.63$ & $3.4 \pm 0.18$ & $3.2 \pm 0.26$ & $6.8 \pm 0.23$ & $2.1 \pm 0.14$ \\
\hline & Other/multiple & $9.1 \pm 0.36$ & $5.7 \pm 0.30$ & $11.2 \pm 0.35$ & $10.1 \pm 0.41$ & $8.9 \pm 0.64$ & $3.8 \pm 0.19$ & $3.4 \pm 0.27$ & $7.9 \pm 0.26$ & $2.5 \pm 0.15$ \\
\hline \multirow[t]{3}{*}{ Marital status } & Never married (ref.) & $8.6 \pm 0.33$ & $5.4 \pm 0.28$ & $10.3 \pm 0.32$ & $9.5 \pm 0.39$ & $8.8 \pm 0.62$ & $3.9 \pm 0.17$ & $3.8 \pm 0.25$ & $6.9 \pm 0.22$ & $2.7 \pm 0.13$ \\
\hline & Currently married & $9.0 \pm 0.32$ & $5.5 \pm 0.27$ & $11.0 \pm 0.31$ & $10.0 \pm 0.38$ & $9.6 \pm 0.62$ & $3.8 \pm 0.15$ & $3.6 \pm 0.23$ & $7.0 \pm 0.20$ & $2.2 \pm 0.12$ \\
\hline & $\begin{array}{l}\text { Separated/divorced/ } \\
\text { widowed }\end{array}$ & $8.8 \pm 0.35$ & $5.2 \pm 0.29$ & $10.4 \pm 0.34$ & $9.4 \pm 0.40$ & $9.0 \pm 0.63$ & $3.7 \pm 0.18$ & $3.9 \pm 0.27$ & $7.5 \pm 0.25$ & $2.5 \pm 0.15$ \\
\hline \multirow[t]{2}{*}{ Children } & 0 children (ref.) & $8.5 \pm 0.32$ & $5.2 \pm 0.27$ & $10.3 \pm 0.31$ & $9.4 \pm 0.38$ & $8.7 \pm 0.62$ & $3.8 \pm 0.15$ & $3.8 \pm 0.23$ & $7.0 \pm 0.20$ & $2.6 \pm 0.12$ \\
\hline & 1+ children & $9.1 \pm 0.33$ & $5.6 \pm 0.28$ & $10.9 \pm 0.32$ & $9.8 \pm 0.39$ & $9.6 \pm 0.62$ & $3.8 \pm 0.16$ & $3.7 \pm 0.24$ & $7.3 \pm 0.22$ & $2.4 \pm 0.13$ \\
\hline \multirow[t]{6}{*}{ Pay grade } & E1-E4 (ref.) & $8.9 \pm 0.14$ & $5.7 \pm 0.12$ & $9.3 \pm 0.13$ & $9.0 \pm 0.13$ & $8.7 \pm 0.13$ & $3.7 \pm 0.10$ & $4.8 \pm 0.13$ & $6.0 \pm 0.15$ & $2.8 \pm 0.08$ \\
\hline & E5-E6 & $8.7 \pm 0.13$ & $5.5 \pm 0.11$ & $9.4 \pm 0.13$ & $8.7 \pm 0.13$ & $8.5 \pm 0.13$ & $3.8 \pm 0.10$ & $4.6 \pm 0.13$ & $7.2 \pm 0.14$ & $2.6 \pm 0.08$ \\
\hline & E7-E9 & $8.5 \pm 0.15$ & $5.2 \pm 0.12$ & $9.3 \pm 0.15$ & $8.4 \pm 0.15$ & $8.2 \pm 0.15$ & $3.8 \pm 0.11$ & $4.0 \pm 0.15$ & $8.1 \pm 0.18$ & $2.6 \pm 0.09$ \\
\hline & W01-WO5 & $8.7 \pm 0.27$ & $5.1 \pm 0.19$ & $8.9 \pm 0.25$ & $8.1 \pm 0.25$ & $8.2 \pm 0.25$ & $3.9 \pm 0.18$ & $3.5 \pm 0.21$ & $8.5 \pm 0.31$ & $2.7 \pm 0.14$ \\
\hline & O1-O3 & $9.3 \pm 0.18$ & $5.1 \pm 0.14$ & $9.5 \pm 0.18$ & $9.0 \pm 0.18$ & $9.0 \pm 0.17$ & $4.1 \pm 0.13$ & $3.8 \pm 0.16$ & $7.2 \pm 0.20$ & $2.5 \pm 0.10$ \\
\hline & O4-010 & $9.3 \pm 0.21$ & $5.2 \pm 0.16$ & $9.2 \pm 0.21$ & $8.6 \pm 0.21$ & $8.8 \pm 0.20$ & $4.7 \pm 0.15$ & $3.6 \pm 0.18$ & $8.4 \pm 0.25$ & $2.7 \pm 0.11$ \\
\hline
\end{tabular}

\footnotetext{
${ }^{a}$ Reference categories are indicated for tests of differences between means. All differences from the reference category are statistically significant $(P<0.05)$, except for means in bold. Linear regressions to estimate least square means were adjusted for sample weights. Multivariate analyses included all demographic characteristics simultaneously
}

current smoking, in fully adjusted models, was associated with higher odds of high blood pressure, low HDL cholesterol, and high triglycerides; only former smoking was associated with higher odds of overweight/obesity. Alcohol intake was also associated with higher odds of high blood pressure and blood glucose (Fig. 1a-f; Additional file 1: Table S4).
Among the 11 dietary factors, across models, lean meats and sweets were associated with lower odds of high blood pressure, dairy with lower odds of high cholesterol, starch with lower odds of high triglycerides, and dairy and lean meat with lower odds of low HDL cholesterol. Intake of whole grains, snacks, sweets, and sugary drinks were associated with significantly lower odds of overweight/obesity, 
Table 4 Odds ratios $(95 \% \mathrm{Cl})$ of medical conditions by sociodemographic characteristics ${ }^{\text {a }}$

\begin{tabular}{|c|c|c|c|c|c|c|c|}
\hline Characteristic & Categories & $\begin{array}{l}\text { High blood } \\
\text { pressure }\end{array}$ & $\begin{array}{l}\text { High } \\
\text { cholesterol }\end{array}$ & $\begin{array}{l}\text { High } \\
\text { triglycerides }\end{array}$ & $\begin{array}{l}\text { Low HDL } \\
\text { cholesterol }\end{array}$ & $\begin{array}{l}\text { High blood } \\
\text { glucose }\end{array}$ & $\begin{array}{l}\text { Overweight/ } \\
\text { obesity }\end{array}$ \\
\hline Cases (N) & & 3713 & 4098 & 1745 & 1921 & 499 & 16,292 \\
\hline Age & $21-25$ yrs & $1.69(1.13-2.53)$ & $1.27(0.67-2.38)$ & $1.25(0.41-3.83)$ & $1.21(0.45-3.25)$ & $1.13(0.34-3.79)$ & $1.54(1.28-1.84)$ \\
\hline \multirow[t]{3}{*}{ (vs. $\leq 20$ yrs) } & $26-35$ yrs & $2.30(1.51-3.50)$ & $2.94(1.57-5.50)$ & $2.59(0.87-7.74)$ & $2.79(1.05-7.38)$ & $1.34(0.40-4.54)$ & $2.21(1.80-2.70)$ \\
\hline & $36-45$ yrs & $3.77(2.43-5.83)$ & $8.12(4.28-15.38)$ & $7.94(2.63-23.93)$ & $3.54(1.04-12.06)$ & $3.82(3.05-4.78)$ & \\
\hline & $46+y r s$ & $6.33(4.01-10.01)$ & $12.54(6.54-24.06)$ & $11.81(3.88-36.00)$ & $11.80(4.37-31.88)$ & $10.06(2.85-35.52)$ & $4.19(3.22-5.45)$ \\
\hline $\begin{array}{l}\text { Service } \\
\text { Branch }\end{array}$ & Air Force & $0.80(0.70-0.91)$ & $0.98(0.86-1.11)$ & $1.26(1.05-1.52)$ & $0.97(0.82-1.15)$ & $0.73(0.52-1.02)$ & $0.79(0.71-0.88)$ \\
\hline \multirow[t]{3}{*}{ (vs. Army) } & $\begin{array}{l}\text { Coast } \\
\text { Guard }\end{array}$ & $0.72(0.62-0.85)$ & $1.24(1.07-1.44)$ & 1.59 (1.29-1.95) & $1.27(1.04-1.54)$ & $1.01(0.68-1.50)$ & $1.05(0.93-1.19)$ \\
\hline & $\begin{array}{l}\text { Marine } \\
\text { Corps }\end{array}$ & $0.98(0.84-1.15)$ & $0.95(0.81-1.11)$ & $0.68(0.52-0.88)$ & $0.66(0.53-0.83)$ & $1.01(0.69-1.50)$ & $0.78(0.69-0.88)$ \\
\hline & Navy & $0.85(0.73-0.99)$ & $1.24(1.07-1.43)$ & $1.57(1.28-1.91)$ & $1.25(1.04-1.51)$ & $1.45(1.05-2.02)$ & $1.04(0.91-1.18)$ \\
\hline $\begin{array}{l}\text { Sex } \\
\text { (vs. male) }\end{array}$ & Female & $0.54(0.49-0.60)$ & $0.65(0.58-0.72)$ & $0.56(0.47-0.66)$ & $0.47(0.40-0.55)$ & 1.35 (1.03-1.77) & $0.29(0.27-0.31)$ \\
\hline Education & $\begin{array}{l}\text { Some } \\
\text { college }\end{array}$ & $0.99(0.87-1.14)$ & $1.15(0.98-1.34)$ & $1.18(0.94-1.48)$ & $1.25(1.00-1.56)$ & $0.95(0.66-1.36)$ & $1.03(0.93-1.14)$ \\
\hline \multirow{3}{*}{$\begin{array}{l}\text { (vs. high } \\
\text { school or } \\
\text { equiv.) }\end{array}$} & $\begin{array}{l}\text { 2-year } \\
\text { college }\end{array}$ & $0.84(0.71-0.99)$ & $1.13(0.95-1.34)$ & $1.33(1.03-1.70)$ & $1.50(1.18-1.91)$ & $1.01(0.67-1.51)$ & $0.88(0.77-1.00)$ \\
\hline & $\begin{array}{l}\text { 4-year } \\
\text { college }\end{array}$ & $0.84(0.69-1.00)$ & $1.19(0.98-1.43)$ & $1.59(1.22-2.08)$ & $1.52(1.18-1.96)$ & $0.62(0.40-0.98)$ & $0.94(0.81-1.09)$ \\
\hline & $\begin{array}{l}\text { Beyond } \\
\text { college }\end{array}$ & $0.89(0.72-1.08)$ & $1.39(1.13-1.71)$ & $1.73(1.30-2.30)$ & $1.92(1.45-2.56)$ & $1.11(0.67-1.82)$ & $0.83(0.70-0.98)$ \\
\hline $\begin{array}{l}\text { Race/ } \\
\text { ethnicity }\end{array}$ & Hispanic & $0.89(0.77-1.03)$ & $1.06(0.91-1.22)$ & $1.04(0.84-1.27)$ & $0.90(0.73-1.10)$ & $1.39(1.00-1.95)$ & $1.43(1.28-1.59)$ \\
\hline \multirow[t]{2}{*}{$\begin{array}{l}\text { (vs. non- } \\
\text { Hispanic } \\
\text { white) }\end{array}$} & $\begin{array}{l}\text { Non- } \\
\text { Hispanic } \\
\text { black }\end{array}$ & $1.68(1.46-1.93)$ & $1.11(0.96-1.30)$ & $0.58(0.44-0.75)$ & $0.78(0.62-0.98)$ & $1.42(0.99-2.05)$ & $1.36(1.20-1.55)$ \\
\hline & $\begin{array}{l}\text { Other/ } \\
\text { multiple }\end{array}$ & $1.43(1.23-1.67)$ & $1.44(1.23-1.67)$ & $1.37(1.12-1.67)$ & $1.20(0.98-1.47)$ & $1.90(1.36-2.66)$ & $0.91(0.80-1.03)$ \\
\hline $\begin{array}{l}\text { Marital } \\
\text { status }\end{array}$ & $\begin{array}{l}\text { Currently } \\
\text { married }\end{array}$ & $1.12(0.97-1.31)$ & $1.26(1.08-1.48)$ & $1.03(0.81-1.30)$ & $0.96(0.76-1.20)$ & $1.11(0.72-1.72)$ & $1.25(1.14-1.38)$ \\
\hline $\begin{array}{l}\text { (vs. never } \\
\text { married) }\end{array}$ & $\begin{array}{l}\text { Separated/ } \\
\text { divorced/ } \\
\text { widowed }\end{array}$ & $1.31(1.10-1.57)$ & $1.18(0.98-1.42)$ & $0.93(0.71-1.23)$ & $0.96(0.74-1.25)$ & $1.42(0.89-2.26)$ & $1.07(0.94-1.21)$ \\
\hline $\begin{array}{l}\text { Children } \\
\text { (vs. none) }\end{array}$ & $1+$ children & $1.06(0.96-1.18)$ & $1.08(0.98-1.20)$ & $1.24(1.08-1.43)$ & $1.32(1.16-1.52)$ & $1.64(1.27-2.11)$ & $1.23(1.13-1.34)$ \\
\hline Pay grade & E5-E6 & $1.57(1.34-1.85)$ & $1.90(1.58-2.28)$ & $2.10(1.60-2.76)$ & $1.86(1.41-2.46)$ & $1.63(1.09-2.45)$ & $1.54(1.39-1.72)$ \\
\hline \multirow[t]{4}{*}{ (vs. E1-E4) } & E7-E9 & $1.68(1.39-2.03)$ & $2.07(1.68-2.55)$ & $2.22(1.64-3.00)$ & $2.13(1.57-2.89)$ & $1.41(0.90-2.21)$ & $1.73(1.50-2.01)$ \\
\hline & W01-WO5 & $1.41(1.10-1.82)$ & $2.28(1.76-2.95)$ & $2.23(1.53-3.24)$ & $2.25(1.55-3.26)$ & $1.00(0.55-1.81)$ & $1.87(1.49-2.35)$ \\
\hline & O1-O3 & $1.12(0.90-1.39)$ & 1.41 (1.13-1.77) & $1.42(1.03-1.97)$ & $1.34(0.96-1.86)$ & $1.05(0.61-1.80)$ & $1.18(1.01-1.37)$ \\
\hline & O4-O10 & $1.15(0.90-1.47)$ & $1.63(1.27-2.09)$ & $1.49(1.05-2.12)$ & $1.58(1.11-2.27)$ & $0.86(0.48-1.54)$ & $1.27(1.04-1.55)$ \\
\hline
\end{tabular}

${ }^{a}$ Logistic regressions were weighted by sample weights. Fully-adjusted models included all sociodemographic characteristics simultaneously, current enrollment in a weight-loss program, history of weight loss, and history of deployment. In bold text are odds ratios that are significantly different from the reference category. For simpler (less adjusted) models, see Additional file 1: Table S3

while caffeinated drinks were associated with higher odds (Fig. 1a-f; Additional file 1: Table S4).

\section{Factor analyses of behaviors and risks}

Exploratory factor analyses conducted found that 4 factors explained $9 \%$ of the variance in the original behavioral variables (Additional file 1: Table S2). They were characterized as "Healthy diet" (based on high loadings of fruit, starch, vegetable, whole grain, dairy, and lean meat intake), "Unhealthy diet" (based on high loadings of starch, snacks, sweets, sugary drinks, fried food, and caffeinated drinks), "Exercise" (based on high loadings of moderate and vigorous aerobic exercise and strength training), and "Bad habits" (based on high loadings of alcohol, smoking, and caffeinated drinks, and inverse loading of sleep). The factors were associated with medical conditions in 
a

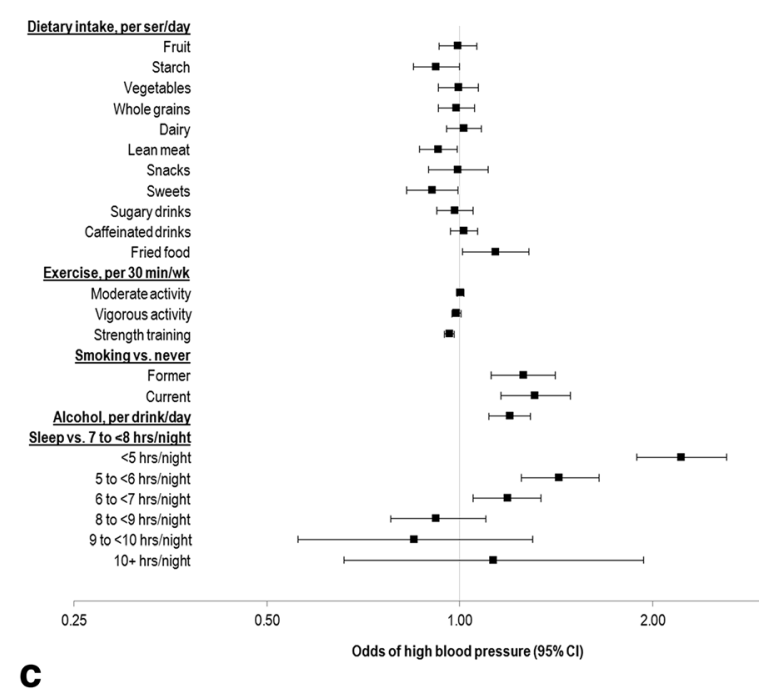

C

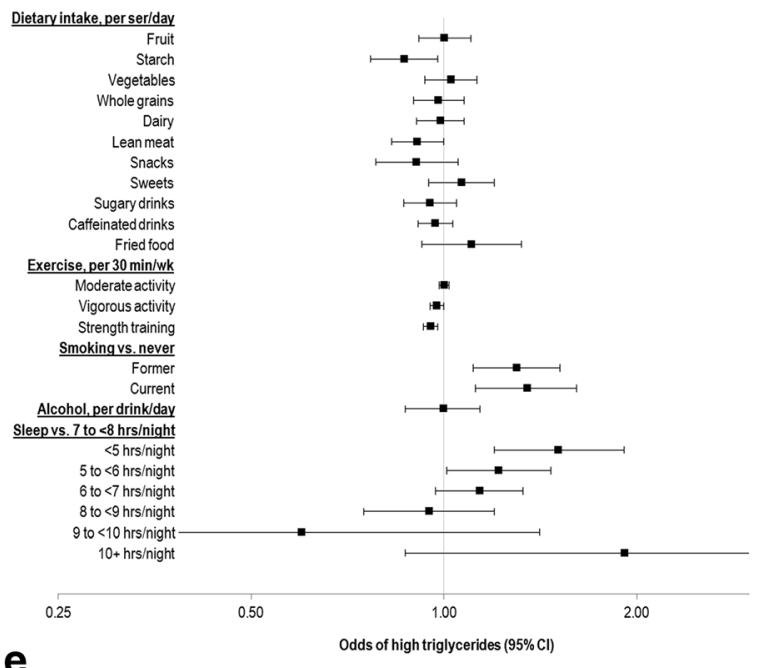

e

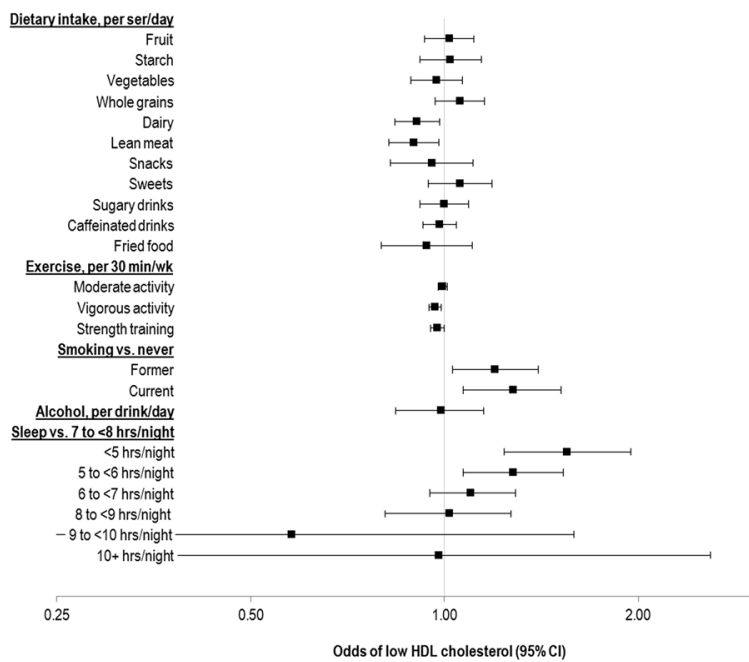

b

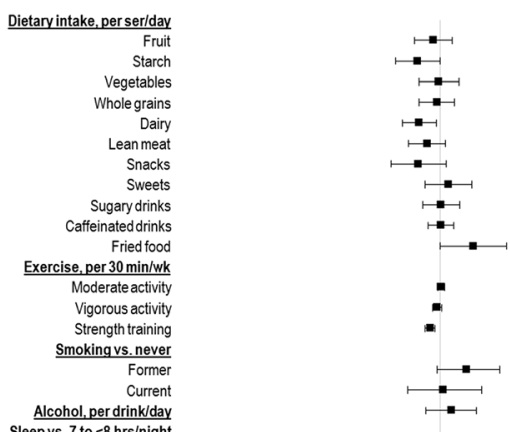

Sleepvs. 7 to $<8$ hrs/night

$<5 \mathrm{hrs} / \mathrm{night}$
5 to $<6 \mathrm{hrs} / \mathrm{night}$

6 to $<7$ hrsinight

8 to $<9$ hrs inight

to $<10$ hrs/night
$10+$ hrs/night

0.25

0.50

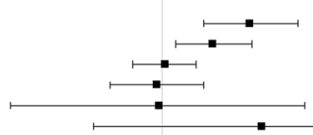

d

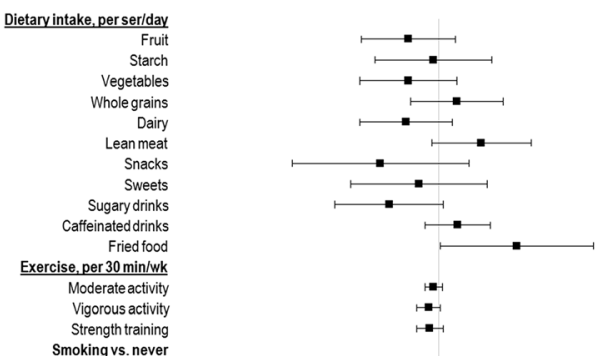

Smokingus. never

Former
Current

Alcohol, per drink/day

$\frac{\text { Sleep vs. } 7 \text { to }<8 \text { hrs/night }}{<5 \mathrm{hrs} \text { night }}$

5 to $<6$ hrs $/$ inght

5 to $<6$ hrssinght
6 to $<7$ hrsinight

8 to $<9$ hrs/night

-9 to $<10$ hrs/night

0.25

f

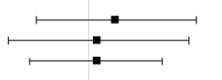

$\frac{\text { Dietary intake, per ser/day }}{\text { Fruit }}$

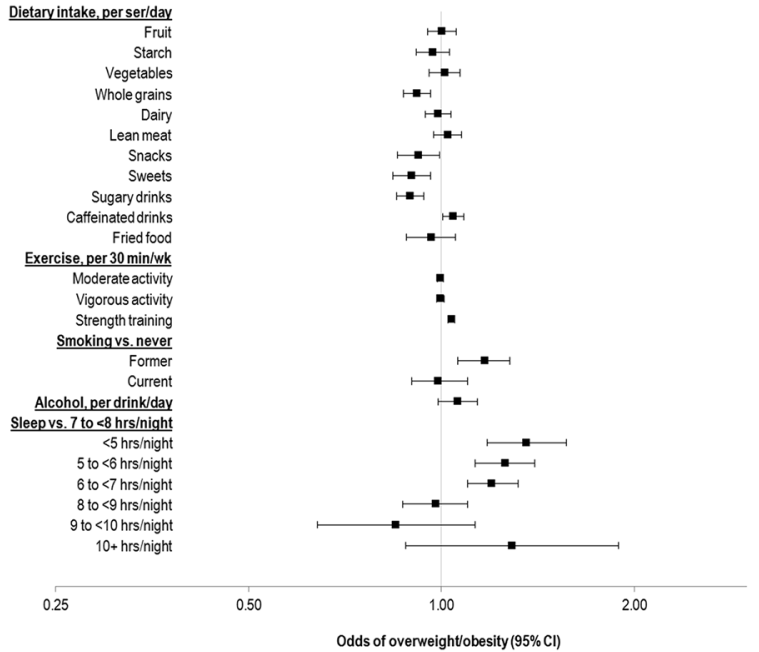

Fig. 1 (See legend on next page.) 
(See figure on previous page.)

Fig. 1 Odds of doctor-informed medical conditions according to health behaviors. Forest plot of odds of having self-reported high blood pressure $(\mathbf{a})$, cholesterol (b), triglycerides (c), blood glucose (d), low high-density lipoprotein [HDL] cholesterol (e), or being overweight/obese $(\mathbf{f})$ by health behaviors. Models were adjusted for all behaviors simultaneously, sociodemographic characteristics (age, sex, service branch, education, race/ethnicity, marital status, children living with the respondent, and pay grade), current enrollment in a weight-loss program, history of weight loss, and history of deployment. Odds ratios are given by the enclosed squares; $95 \%$ confidence intervals are given by the bars. For related data, see Additional file 1: Table S4

generally expected ways: higher "Healthy diet" was consistently inversely associated with odds of high blood pressure, high cholesterol, high triglycerides, low HDL cholesterol, and overweight/obesity (Table 5). Higher "Unhealthy diet" was only consistently associated with lower odds of overweight/obesity. "Exercise" was consistently associated with lower odds of high cholesterol, high triglycerides, high blood glucose, and low HDL cholesterol, and was associated with higher odds overweight/obesity. Finally, "Bad habits" was strongly

Table 5 Odds ratios $(95 \% \mathrm{Cl})$ of medical conditions by derived behavioral factors ${ }^{\text {a }}$

\begin{tabular}{|c|c|c|c|c|}
\hline Factor & Name & Model 1 & Model 2 & Model 3 \\
\hline & & \multicolumn{3}{|c|}{ Odds of high blood pressure $(\mathrm{N}$ cases $=3713$ ) } \\
\hline 1 & "Healthy diet" & $0.86(0.81-0.90)$ & $0.88(0.83-0.93)$ & $0.87(0.83-0.93)$ \\
\hline 2 & "Unhealthy diet" & $0.89(0.83-0.94)$ & $0.96(0.90-1.02)$ & $0.97(0.91-1.03)$ \\
\hline 3 & "Exercise" & $0.89(0.84-0.94)$ & $0.95(0.90-1.01)$ & $0.95(0.89-1.01)$ \\
\hline \multirow[t]{2}{*}{4} & "Bad habits" & $1.40(1.34-1.48)$ & $1.41(1.33-1.49)$ & $1.39(1.31-1.48)$ \\
\hline & & \multicolumn{3}{|c|}{ Odds of high cholesterol ( $\mathrm{N}$ cases $=4098)$} \\
\hline 1 & "Healthy diet" & $0.87(0.82-0.91)$ & $0.83(0.79-0.88)$ & $0.83(0.79-0.88)$ \\
\hline 2 & "Unhealthy diet" & $0.83(0.78-0.88)$ & $1.00(0.93-1.06)$ & $1.00(0.94-1.07)$ \\
\hline 3 & "Exercise" & $0.70(0.66-0.74)$ & $0.85(0.80-0.90)$ & $0.85(0.80-0.90)$ \\
\hline \multirow[t]{2}{*}{4} & "Bad habits" & $1.08(1.03-1.13)$ & $1.10(1.04-1.17)$ & $1.10(1.03-1.17)$ \\
\hline & & \multicolumn{3}{|c|}{ Odds of high triglycerides $(\mathrm{N}$ cases $=1745)$} \\
\hline 1 & "Healthy diet" & $0.89(0.83-0.96)$ & $0.83(0.77-0.90)$ & $0.83(0.77-0.90)$ \\
\hline 2 & "Unhealthy diet" & $0.77(0.71-0.84)$ & $0.93(0.85-1.02)$ & $0.94(0.85-1.03)$ \\
\hline 3 & "Exercise" & $0.61(0.56-0.67)$ & $0.79(0.72-0.86)$ & $0.78(0.71-0.85)$ \\
\hline \multirow[t]{2}{*}{4} & "Bad habits" & $1.11(1.04-1.18)$ & $1.16(1.07-1.26)$ & $1.15(1.06-1.26)$ \\
\hline & & \multicolumn{3}{|c|}{ High blood glucose $(\mathrm{N}$ cases $=499)$} \\
\hline 1 & "Healthy diet" & $0.85(0.73-0.99)$ & $0.87(0.76-1.01)$ & $0.87(0.75-1.00)$ \\
\hline 2 & "Unhealthy diet" & $0.72(0.61-0.86)$ & $0.85(0.72-1.00)$ & $0.87(0.74-1.03)$ \\
\hline 3 & "Exercise" & $0.64(0.52-0.77)$ & $0.75(0.62-0.91)$ & $0.74(0.61-0.90)$ \\
\hline \multirow[t]{2}{*}{4} & "Bad habits" & $1.21(1.08-1.36)$ & $1.25(1.09-1.44)$ & $1.25(1.08-1.44)$ \\
\hline & & \multicolumn{3}{|c|}{ Low HDL cholesterol $(\mathrm{N}$ cases $=1921)$} \\
\hline 1 & "Healthy diet" & $0.91(0.85-0.98)$ & $0.86(0.80-0.93)$ & $0.86(0.80-0.93)$ \\
\hline 2 & "Unhealthy diet" & $0.81(0.75-0.88)$ & $0.97(0.89-1.06)$ & $0.98(0.90-1.07)$ \\
\hline 3 & "Exercise" & $0.63(0.58-0.69)$ & $0.79(0.72-0.87)$ & $0.79(0.72-0.86)$ \\
\hline \multirow[t]{2}{*}{4} & "Bad habits" & $1.11(1.04-1.19)$ & $1.16(1.07-1.27)$ & $1.16(1.06-1.26)$ \\
\hline & & \multicolumn{3}{|c|}{ Odds of overweight/obesity ( $\mathrm{N}$ cases $=16,292$ ) } \\
\hline 1 & "Healthy diet" & $0.94(0.91-0.98)$ & $0.94(0.91-0.99)$ & $0.95(0.91-0.99)$ \\
\hline 2 & "Unhealthy diet" & $0.75(0.72-0.78)$ & $0.78(0.74-0.81)$ & $0.80(0.76-0.83)$ \\
\hline 3 & "Exercise" & $1.05(1.01-1.09)$ & $1.11(1.06-1.16)$ & $1.12(1.07-1.18)$ \\
\hline 4 & "Bad habits" & $1.20(1.15-1.25)$ & $1.10(1.05-1.16)$ & $1.09(1.04-1.15)$ \\
\hline
\end{tabular}


associated with higher odds of high blood pressure, high cholesterol, high triglycerides, high blood glucose, low HDL cholesterol, and overweight/obesity.

\section{Discussion}

We used a large anonymous self-reported survey of the Armed Forces to assess relationships between sociodemographic characteristics, health behaviors, and doctor-informed medical conditions and overweight/ obesity. When we examined individual health behaviors and medical conditions, by far the most consistent and largest relationship was between low sleep duration and higher odds of every medical condition. Otherwise, other behaviors, including dietary and exercise behaviors, were inconsistently associated with medical conditions. Age, sex, marital status, and presence of children appeared to be the only relatively consistent sociodemographic factors related to health behaviors. Older individuals, females, and those residing with children tended to exercise less, but eat healthier foods and not smoke. Older individuals also slept less, as did those with children present. Those who were married appeared to have consumed a better diet, exercised more, and slept more than their non-married counterparts. In terms of medical conditions, older age and female sex appeared to be the only consistent drivers of higher and lower odds, respectively, of the presence of a given medical condition. Relationships of race/ethnicity, education, service branch, and marital status with medical conditions were inconsistent. Finally, in exploratory factor analyses of health behaviors, associations of behavioral patternstwo patterns in particular-with medical conditions were associated with medical conditions as expected: the pattern characterized by a "healthy" diet was associated with lower odds of all medical conditions except high blood pressure, while the pattern characterized by "bad" habits (including lack of sleep) was associated with higher odds of every medical condition.

We observed robust associations between short sleep and each medical condition in the present study, ranging from $36 \%$ higher odds of overweight/obesity to over double the odds of hypertension, in contrast to inconsistent findings for diet and exercise, and smoking. There is increasing evidence of the importance of sleep to health [17-23], for example, a 2015 meta-analysis of 10 prospective cohort studies investigating sleep in relation to type 2 diabetes risk reported a U-shaped, dose-response relationship between sleep duration and diabetes risk [22]. The lowest risk was observed at sleep durations of 7-8 h/day, with incrementally higher risk of diabetes below or above that duration. Several reports have linked short sleep durations with higher risk of overweight/obesity [17, 20,21], including an analysis of the representative U.S. population, in which authors reported that, relative to those who slept 7-8 h/ day, very short sleepers $(<5 \mathrm{~h})$ had $30 \%$ higher odds of being overweight and were twice as likely to be obese, while (5-6 h) short sleepers had 20\% higher odds of being overweight and 57\% higher odds of being obese [20]. In addition, a 2013 meta-analysis of 17 cohorts investigating the relationship between sleep duration and incident hypertension reported that short sleep duration conferred $21 \%$ higher risk of developing the condition [18]. Because recommendations to change diet, exercise, and smoking behaviors, but not sleep behaviors, are a part of many primary and secondary prevention recommendations [24-26], it is possible that individuals are more likely to make changes to these behaviors, rather than sleep, in an effort to prevent or as a result of receiving a diagnosis. In fact, sleep is not mentioned as a modifiable risk factor in disease prevention/management guidelines from the American Diabetes Association or from the American College of Cardiology/American Heart Association [24-26], and thus may not be a part of lifestyle changes individuals undertake to modify their perceived disease risk.

In military populations, the critical importance of sleep has only recently become part of the "Performance Triad", an educational initiative introduced in 2013 on health readiness and wellness including 1 ) physical activity; 2) nutrition; and 3) sleep [27, 28]. Our data therefore confirm the importance of the inclusion of sleep in the "Triad" initiative. In our study, poor sleep may also be a complex product of poor health, and occupational or personal stress $[29,30]$. Other studies have indicated that sleep disorders, including insomnia and sleep apnea, appear to be more common in service members than in civilians [31-33]. Our observations using self-reported survey data, on the strong relationship between poor sleep and behaviors and medical conditions, agree with other studies usingself-reported surveys of military personnel [34].

Our observations regarding select sociodemographic characteristics, notably age, sex, and marital status, as being associated with select health behaviors generally agree with existing evidence $[2,7]$. With the exception of odds of high blood glucose, which was higher in females versus males, other associations of age and sex-well-known biological drivers of health risks-were as generally expected. Married individuals reported better health behaviors than never- or previously married individuals; however this did not necessarily translate into lower odds of having medical conditions for married individuals. Given the cross-sectional nature of the study design, we can only speculate as to why: first, married individuals may be more likely to seek routine medical care at the urging of their spouse [35-37], and thus have been told by a physician of a prevalent condition that would have otherwise gone undetected. Second, cross-sectional analyses preclude assessment of sequential behaviors; the reported behaviors may be in response to a diagnosis, a 
form of reverse causality. In addition, although analyses were adjusted for age and presence of children, as well as other sociodemographic characteristics, relationships between marital status and medical conditions are nuanced and complicated by time-related factors, including secular trends [37-39]. For example, a recent study using data from a panel of nationally representative households observed that while a protective association of long marriage on women's self-reported health was evident in the earlier birth cohort (born 1955-1964), it had reversed in the most recent birth cohort (born 1975-1984) [39].

There were other unexpected associations. For example, we observed intake of snacks, sweets, and sugary drinks (in addition to the "Unhealthy diet" factor which included these foods) were associated with lower odds of overweight/obesity, which is generally the opposite of what would be expected given evidence regarding the long-term negative consequences of consuming these foods on health [40-43]. These associations held despite adjusting for history of weight loss and current enrollment in a weight control program. It is possible this finding reflects the generally young age of the respondents in this military population, or that some respondents were simply not at risk of overweight. This observation, in fact, appears to be consistent with cross-sectional nationally representative NHANES data on snacking and sugary beverages in both adolescents [44] and adults [45] which have shown no clearly discernible associations with overweight or obesity. In addition, state-based BRFSS 2013 data indicate that sugar-sweetened beverage intake declines with higher age. Intake was most prevalent among younger adults (age 18-24 y), at 43\% reporting consuming these beverages one or more times per day, while those in older age groups reported less consumption (38\% of those age 25$34 \mathrm{y}, 30 \%$ in those age $35-54 \mathrm{y}$, and $19 \%$ in those age $\geq$ 55 y) [46]. On the other hand, our exploratory observations regarding the "healthy diet" pattern and favorable odds of medical conditions were consistent with dietary pattern literature [47-50].

As noted above, our analyses are limited by the cross-sectional nature of the survey, which precludes causal inferences. Particularly when assessing health behaviors and medical conditions concurrently, a behavioral pattern may reflect a subset of respondents who have changed their behaviors as result of having been alerted to a health risk [51-56]. Furthermore, we were limited to gross rather than granular assessments of dietary components. We were also likely limited in the validity of our inferences by social desirability response bias of self-reported data. In addition, non-response bias, length of the survey, and fear of loss of anonymity among either non-respondents or due to selective non-responses in those excluded from the present analysis may also limit our ability to make inferences or generalize our findings to the broader Armed Services, despite using revised sampling weights to accommodate exclusions.

\section{Conclusions}

This study presents relationships between sociodemographic characteristics, health behaviors, and doctor-informed medical conditions and overweight/obesity in the U.S. Armed Forces, which are typically not included, or not separately reported, in national or state health surveys.

Despite aforementioned limitations and our inability to deduce causal relationships, we observed pronounced associations between health behaviors-especially sleep duration - and medical conditions, thus adding to evidence that sleep is a critical, potentially modifiable behavior within this population. It is recognized that there are times when sleep restriction is unavoidable in this population (e.g., during periodic training and mission scenarios), however, adequate sleep should continue to be promoted as an important part of overall health and wellness throughout the military community.

\section{Additional file}

\begin{abstract}
Additional file 1: Table S1. Unweighted, unadjusted characteristics of respondents who were included vs. excluded in the present analysis, from the 2011 Health Related Behaviors Survey. Table S2. Factors and factor loadings of 17 health behaviors derived from principal components analysis with varimax rotation. Table S3. Odds ratios (95\% Cl) of medical conditions by sociodemographic characteristics. Table S4. Description of Data: Odds ratios $(95 \% \mathrm{Cl})$ of medical conditions by health behaviors. (DOCX $50 \mathrm{~kb}$ )
\end{abstract}

\section{Abbreviations}

BMI: Body mass index; Cl: Confidence interval; d: Day; DOD: Department of Defense; HDL: High-density lipoprotein; HRBS: Health Related Behavior Survey; hrs: Hours; OR: Odds ratio; ser: Servings; TMA: Tricare Management Activity; U.S.: United States; USCG: United States Coast Guard; wk.: Week; y: Years

\section{Acknowledgements}

The authors would like to thank members of the US Armed Forces and US Coast Guard who responded to the 2011 HRBS. The opinions or assertions contained herein are the private views of the authors and are not to be construed as official or reflecting the views of the US Department of Defense or US Coast Guard. Any citations of commercial organizations and trade names in this report do not constitute an official endorsement of approval of the products or services of these organizations. Dr. Hruby's contribution to this research was supported by an appointment to the Postgraduate Research Participation Program at the US Army Medical Research Institute of Environmental Medicine administered by the Oak Ridge Institute for Science and Education through an interagency agreement between the US Department of Energy and USAMRMC.

\section{Funding}

This work was supported by the US Army Medical Research and Materiel Command.

\section{Availability of data and materials}

Authors obtained the HRBS dataset supporting the conclusions of this article via a data use agreement with Tricare Management Activity. The data that support the findings of this study are available from Tricare Management 
Activity but restrictions apply to the availability of these data, which were used under license for the current study, and are therefore not publicly available. However, data are available to interested parties upon reasonable request and with permission of Tricare Management Activity.

\section{Authors' contributions}

TJS conceived of and designed the study, and revised the article for important intellectual content. AH conducted the analyses and drafted the manuscript. HRL revised the article for important intellectual content. All authors reviewed, edited, and made important intellectual contributions to the manuscript. TJS and AH had full access to all the data in the study and take full responsibility for the integrity of the data and the accuracy of the data analysis. All authors read and approved the final manuscript.

\section{Ethics approval and consent to participate}

The 2011 HRBS was conducted by ICF International under advisement of the Office of the Assistant Secretary of Defense for Health Affairs, Tricare Management Activity (TMA; now Defense Health Agency), and the USCG [15]. Primary data collection was approved by the Office of the Assistant Secretary of Defense for Health Affairs/TRICARE Management Activity (OASD(HA)/TMA), Human Research Protection Office. Informed consent was required and obtained the beginning of the HRBS, and responses were anonymous. For the present secondary data analyses, we obtained a de-identified data file from TMA through a data use agreement. Because the data were previously collected and de-identified, the protocol for the present study was deemed exempt (not human subject research) by both the US Army Research Institute of Environmental Medicine (Natick, MA) and the Office of the Assistant Secretary of Defense for Health Affairs/TRICARE Management Activity (OASD(HA)/TMA), Human Research Protection Office.

\section{Consent for publication}

Not applicable (no individual-level data included).

\section{Competing interests}

The authors declare that they have no competing interests.

\section{Publisher's Note}

Springer Nature remains neutral with regard to jurisdictional claims in published maps and institutional affiliations.

\section{Author details}

${ }^{1}$ Military Nutrition Division, US Army Research Institute of Environmental Medicine, Natick, MA 01760, USA. ${ }^{2}$ Nutritional Epidemiology Program, Jean Mayer USDA Human Nutrition Research Center on Aging at Tufts University, Boston, MA 02111, USA

\section{Received: 27 October 2017 Accepted: 28 June 2018}

\section{Published online: 11 July 2018}

\section{References}

1. Survey of Health Related Behaviors [Internet]. Military Health System. [cited 2016 Nov 16]. Available from: https://health.mil/Military-Health-Topics/ Access-Cost-Quality-and-Safety/Health-Care-Program-Evaluation/Survey-ofHealth-Related-Behaviors/2011-Health-Related-Behavior-Survey-Active-Duty.

2. Smith TJ, Dotson LE, Young AJ, White A, Hadden L, Bathalon GP, Funderburk L, Marriott BP. Eating patterns and leisure-time exercise among active duty military personnel: comparison to the healthy people objectives. J Acad Nutr Diet. 2013;113(7):907-19.

3. Ramsawh HJ, Fullerton CS, Mash HBH, Ng THH, Kessler RC, Stein MB, Ursano RJ. Risk for suicidal behaviors associated with PTSD, depression, and their comorbidity in the U.S. Army. J Affect Disord. 2014;161:116-22.

4. Lane ME, Hourani LL, Bray RM, Williams J. Prevalence of perceived stress and mental health indicators among reserve-component and active-duty military personnel. Am J Public Health. 2012;102(6):1213-20.

5. Smith TJ, White A, Hadden L, Young AJ, Marriott BP. Associations between mental health disorders and body mass index among military personnel. Am J Health Behav. 2014;38(4):529-40.

6. Reyes-Guzman CM, Bray RM, Forman-Hoffman VL, Williams J. Overweight and Obesity trends among active duty military personnel: a 13-year perspective. Am J Prev Med. 2015;48(2):145-53.
7. Smith TJ, Marriott BP, Dotson L, Bathalon GP, Funderburk L, White A, Hadden L, Young AJ. Overweight and obesity in military personnel: sociodemographic predictors. Obes Silver Spring Md. 2012;20(7):1534-8.

8. Di Nola GM. Stressors afflicting families during military deployment. Mil Med. 2008;173(5):v-vii.

9. Hosek J, MacDermid Wadsworth S. Economic conditions of military families. Futur Child. 2013;23(2):41-59.

10. U.S. Department of the Army. Army Regulation 40-501: Standards of Medical Fitness [Internet]. U.S. Department of the Army, Washington, D.C.; [cited 2014 Apr 1]. Available from: https://armypubs.army.mil/epubs/DR_pubs/DR_ a/pdf/web/ARN3801_AR40-501_Web_FINAL.pdf.

11. U.S. Department of Health and Human Services and U.S. Department of the Army. Field Manual 7-22: Army Physical Readiness Training [Internet]. 2012. Available from: https://quefabsancse.files.wordpress.com/2015/08/us-armyphysical-readiness-training-manual-pdf.pdf.

12. U.S. Department of the Army. Army Regulation 350-1: Army Training and Leader Development [Internet]. 2014. Available from: http://www.dami.army. pentagon.mil/g2Docs/Foundry/r350_1.pdf.

13. Redmond S, Wilcox S, Campbell S, Kim A, Finney K, Barr K, Hassan A. A brief introduction to the military workplace culture. Work. 2015;1:9-20.

14. Adler $A B$, Castro $C A$. An occupational mental health model for the military. Mil Behav Health. 2013;1(1):41-5.

15. Barlas FM, Higgins WB, Pflieger JC, Diecker K. 2011 Department of Defense Health Related Behaviors Survey of active duty military personnel [internet]. Fairfax: ICF international; 2013. Available from: http://www.health.mil/Reference-Center/Reports/2013/02/01/2011-HealthRelated-Behaviors-Active-Duty-Executive-Summary.

16. 2011 Health Related Behaviors Survey of Active Duty Military Personnel Questionnaire [Internet]. [cited 2016 Nov 16]. Available from: https://www. health.mil/Military-Health-Topics/Access-Cost-Quality-and-Safety/HealthCare-Program-Evaluation/Survey-of-Health-Related-Behaviors/2011-HealthRelated-Behavior-Survey-Active-Duty.

17. Lyytikäinen P, Rahkonen O, Lahelma E, Lallukka T. Association of sleep duration with weight and weight gain: a prospective follow-up study. J Sleep Res. 2011;20(2):298-302.

18. Meng $L$, Zheng $Y$, Hui $R$. The relationship of sleep duration and insomnia to risk of hypertension incidence: a meta-analysis of prospective cohort studies. Hypertens res off J Jpn Soc. Hypertens. 2013;36(11):985-95.

19. Nedeltcheva AV, Scheer FAJL. Metabolic effects of sleep disruption, links to obesity and diabetes. Curr Opin Endocrinol Diabetes Obes. 2014;21(4):293-8.

20. Jean-Louis G, Williams NJ, Sarpong D, Pandey A, Youngstedt S, Zizi F, Ogedegbe G. Associations between inadequate sleep and obesity in the US adult population: analysis of the national health interview survey (19772009). BMC Public Health. 2014;14:290.

21. Ford ES, Li C, Wheaton AG, Chapman DP, Perry GS, Croft JB. Sleep duration and body mass index and waist circumference among U.S. adults. Obes Silver Spring Md. 2014;22(2):598-607.

22. Shan Z, Ma H, Xie M, Yan P, Guo Y, Bao W, Rong Y, Jackson CL, Hu FB, Liu L. Sleep duration and risk of type 2 diabetes: a meta-analysis of prospective studies. Diabetes Care. 2015;38(3):529-37.

23. McHill AW, Wright KP. Role of sleep and circadian disruption on energy expenditure and in metabolic predisposition to human obesity and metabolic disease. Obes Rev Off J Int Assoc Study Obes. 2017;18(Suppl 1):15-24.

24. American Diabetes Association. Standards of Medical Care in Diabetes2016. 4. Prevention or Delay of Type 2 Diabetes. Diabetes Care. 2016; 39(Supplement 1):S36-8.

25. American Diabetes Association. 4. Foundations of care: education, nutrition, physical activity, smoking cessation, psychosocial care, and immunization. Diabetes Care. 2015;38(Supplement_1):S20-30.

26. Eckel RH, Jakicic JM, Ard JD, de Jesus JM, Miller NH, Hubbard VS, Lee I-M, Lichtenstein AH, Loria CM, Millen BE, et al. 2013 AHA/ACC guideline on lifestyle management to reduce cardiovascular risk: a report of the American College of Cardiology/American Heart Association task force on practice guidelines. Circ. 2014;129(25 suppl 2):S76-99.

27. Abdullah SP. Performance Triad to Lead Army Medicine to System for Health [Internet]. 2013 [cited 2017 Mar 7]. Available from: https://www.army.mil/article/ 93893/Performance_Triad_to_Lead_Army_Medicine_to_System_for_Health/.

28. Performance Triad [Internet]. [cited 2017 Mar 7]. Available from: https://p3. amedd.army.mil/.

29. Pruiksma KE, Taylor DJ, Wachen JS, Mintz J, Young-McCaughan S, Peterson AL, Yarvis JS, Borah EV, Dondanville KA, Litz BT, et al. Residual sleep 
disturbances following PTSD treatment in active duty military personnel. Psychol Trauma Theory Res Pract Policy. 2016;8(6):697-701.

30. Hunt EJF, Greenberg N, Jones N. Poor sleep after military deployment: associations with mental health difficulties. Occup Med. 2016;8(66):669-75.

31. Hoge CW, McGurk D, Thomas JL, Cox AL, Engel CC, Castro CA. Mild traumatic brain injury in U.S. soldiers returning from Iraq. N Engl J Med. 2008;358(5):453-63.

32. Roth T, Coulouvrat C, Hajak G, Lakoma MD, Sampson NA, Shahly V, Shillington AC, Stephenson JJ, Walsh JK, Kessler RC. Prevalence and Perceived health associated with insomnia based on DSM-IV-TR; international statistical classification of diseases and related health problems, tenth revision; and research diagnostic criteria/international classification of sleep disorders, second edition criteria: results from the America insomnia survey. Biol Psychiatry. 2011;69(6):592-600.

33. Mysliwiec V, Gill J, Lee H, Baxter T, Pierce R, Barr TL, Krakow B, Roth BJ. Sleep disorders in US military personnel: a high rate of comorbid insomnia and obstructive sleep apnea. Chest. 2013;144(2):549-57.

34. Lentino CV, Purvis DL, Murphy KJ, Deuster PA. Sleep as a component of the performance triad: the importance of sleep in a military population. US Army Med Dep J. 2013. p. 98-108. http://www.cs.amedd.army.mil/ FileDownloadpublic.aspx?docid=565febfe-b26e-4922-8f82-0e9373b5f01a.

35. Hanske J, Meyer CP, Sammon JD, Choueiri TK, Menon M, Lipsitz SR, Noldus J, Nguyen PL, Sun M, Trinh Q-D. The influence of marital status on the use of breast, cervical, and colorectal cancer screening. Prev Med. 2016;89:140-5.

36. Cornelis MC, Chiuve SE, Glymour MM, Chang S-C, Tchetgen Tchetgen EJ, Liang L, Koenen KC, Rimm EB, Kawachi I, Kubzansky LD. Bachelors, divorcees, and widowers: does marriage protect men from type 2 diabetes? PLoS One. 2014;9(9):e106720.

37. Dupre ME, Beck AN, Meadows SO. Marital trajectories and mortality among US adults. Am J Epidemiol. 2009;170(5):546-55.

38. Zheng H, Thomas PA. Marital status, self-rated health, and mortality: overestimation of health or diminishing protection of marriage? J Health Soc Behav. 2013;54(1):128-43.

39. Tumin D. Does marriage protect health? A birth cohort comparison*: does marriage protect health? Soc Sci Q. 2018;99(2):626-43.

40. de Koning L, Malik VS, Rimm EB, Willett WC, Hu FB. Sugar-sweetened and artificially sweetened beverage consumption and risk of type 2 diabetes in men. Am J Clin Nutr. 2011;93(6):1321-7.

41. Malik VS, Pan A, Willett WC, Hu FB. Sugar-sweetened beverages and weight gain in children and adults: a systematic review and meta-analysis. Am J Clin Nutr. 2013:98(4):1084-102.

42. Mozaffarian D, Hao T, Rimm EB, Willett WC, Hu FB. Changes in diet and lifestyle and long-term weight gain in women and men. N Engl J Med. 2011;364(25):2392-404.

43. Ma J, Jacques PF, Meigs JB, Fox CS, Rogers GT, Smith CE, Hruby A, Saltzman E, McKeown NM. Sugar-sweetened beverage but not diet soda consumption is positively associated with progression of insulin resistance and prediabetes. J Nutr. 2016;146(12):2544-50.

44. Bleich SN, Wolfson JA. Trends in SSBs and snack consumption among children by age, body weight, and race/ethnicity: snacking among SSB and non-SSB drinkers. Obesity. 2015;23(5):1039-46.

45. Nicklas TA, O'Neil CE, Fulgoni VL III. Snacking patterns, diet quality, and cardiovascular risk factors in adults. BMC Public Health [Internet]. 2014; Dec [cited 2018 Jun 21];14(1). Available from: http://bmcpublichealth. biomedcentral.com/articles/10.1186/1471-2458-14-388.

46. Park S, Xu F, Town M, Blanck HM. Prevalence of sugar-sweetened beverage intake among adults -23 states and the District of Columbia, 2013. MMWR Morb Mortal Wkly Rep. 2016;65(7):169-74.

47. Purvis DL, Lentino CV, Jackson TK, Murphy KJ, Deuster PA. Nutrition as a component of the performance triad: how healthy eating behaviors contribute to soldier performance and military readiness. US Army Med Dep J. 2013. p. 66-78. http://www.cs.amedd.army.mil/FileDownloadpublic. aspx?docid=565febfe-b26e-4922-8f82-0e9373b5f01a.

48. Ley SH, Pan A, Li Y, Manson JE, Willett WC, Sun Q, Hu FB. Changes in overall diet quality and subsequent type 2 diabetes risk: three U.S. prospective cohorts. Diabetes Care. 2016;39(11):2011-8.

49. McEvoy CT, Cardwell CR, Woodside JV, Young IS, Hunter SJ, McKinley MC. A posteriori dietary patterns are related to risk of type 2 diabetes: findings from a systematic review and meta-analysis. J Acad Nutr Diet. 2014;114(11):1759-1775.e4.

50. Nettleton JA, Steffen LM, Schulze MB, Jenny NS, Barr RG, Bertoni AG, Jacobs DR. Associations between markers of subclinical atherosclerosis and dietary patterns derived by principal components analysis and reduced rank regression in the multi-ethnic study of atherosclerosis (MESA). Am J Clin Nutr. 2007;85(6):1615-25.

51. Hawkes AL, Lynch BM, Youlden DR, Owen N, Aitken JF. Health behaviors of Australian colorectal cancer survivors, compared with noncancer population controls. Support Care Cancer. 2008;16(10):1097-104.

52. Patterson RE, Neuhouser ML, Hedderson MM, Schwartz SM, Standish L, Bowen DJ. Changes in diet, physical activity, and supplement use among adults diagnosed with cancer. J Am Diet Assoc. 2003;103(3):323-8.

53. Satia JA, Campbell MK, Galanko JA, James A, Carr C, Sandler RS. Longitudinal changes in lifestyle behaviors and health status in colon cancer survivors. Cancer Epidemiol biomark Prev Publ am Assoc Cancer res cosponsored am Soc Prev. Oncologia. 2004;13(6):1022-31.

54. Keenan PS. Smoking and weight change after new health diagnoses in older adults. Arch Intern Med. 2009:169(3):237.

55. Twardella D, Loew M, Rothenbacher D, Stegmaier C, Ziegler H, Brenner $\mathrm{H}$. The diagnosis of a smoking-related disease is a prominent trigger for smoking cessation in a retrospective cohort study. J Clin Epidemiol. 2006:59(1):82-9.

56. van Gool CH, Kempen GIJM, Penninx BWJH, Deeg DJH, van Eijk JTM. Chronic disease and lifestyle transitions: results from the longitudinal aging study Amsterdam. J Aging Health. 2007;19(3):416-38.

\section{Ready to submit your research? Choose BMC and benefit from:}

- fast, convenient online submission

- thorough peer review by experienced researchers in your field

- rapid publication on acceptance

- support for research data, including large and complex data types

- gold Open Access which fosters wider collaboration and increased citations

- maximum visibility for your research: over $100 \mathrm{M}$ website views per year

At BMC, research is always in progress.

Learn more biomedcentral.com/submissions 